Review

\title{
Chinese Herbal Medicine for the Treatment of Depression: Effects on the Neuroendocrine-Immune Network
}

\author{
Chan $\mathrm{Li}^{1,2}$, Bishan Huang ${ }^{1}$ and Yuan-Wei Zhang ${ }^{1,3, *}$ \\ 1 School of Life Sciences, Guangzhou University, Guangzhou 510006, China; lichan@gzhu.edu.cn (C.L.); \\ 2111914032@e.gzhu.edu.cn (B.H.) \\ 2 Department of Psychiatry, School of Medicine Yale University, New Haven, CT 06511, USA \\ 3 Department of Pharmacology, School of Medicine Yale University, New Haven, CT 06511, USA \\ * Correspondence: yuanwei.zhang@gzhu.edu.cn
}

check for updates

Citation: Li, C.; Huang, B.; Zhang, Y.-W. Chinese Herbal Medicine for the Treatment of Depression: Effects on the Neuroendocrine-Immune

Network. Pharmaceuticals 2021, 14, 65 https://doi.org/10.3390/ph14010065

Received: 1 December 2020

Accepted: 11 January 2021

Published: 14 January 2021

Publisher's Note: MDPI stays neutral with regard to jurisdictional clai$\mathrm{ms}$ in published maps and institutional affiliations.

Copyright: $(\odot 2021$ by the authors. Licensee MDPI, Basel, Switzerland. This article is an open access article distributed under the terms and conditions of the Creative Commons Attribution (CC BY) license (https:// creativecommons.org/licenses/by/ $4.0 /)$.

\begin{abstract}
The neuroimmune and neuroendocrine systems are two critical biological systems in the pathogenesis of depression. Clinical and preclinical studies have demonstrated that the activation of the neuroinflammatory response of the immune system and hyperactivity of the hypothalamuspituitary-adrenal (HPA) axis of the neuroendocrine system commonly coexist in patients with depression and that these two systems bidirectionally regulate one another through neural, immunological, and humoral intersystem interactions. The neuroendocrine-immune network poses difficulties associated with the development of antidepressant agents directed toward these biological systems for the effective treatment of depression. On the other hand, multidrug and multitarget Chinese Herbal Medicine (CHM) has great potential to assist in the development of novel medications for the systematic pharmacotherapy of depression. In this narrative essay, we conclusively analyze the mechanisms of action of $\mathrm{CHM}$ antidepressant constituents and formulas, specifically through the modulation of the neuroendocrine-immune network, by reviewing recent preclinical studies conducted using depressive animal models. Some CHM herbal constituents and formulas are highlighted as examples, and their mechanisms of action at both the molecular and systems levels are discussed. Furthermore, we discuss the crosstalk of these two biological systems and the systems pharmacology approach for understanding the system-wide mechanism of action of $\mathrm{CHM}$ on the neuroendocrine-immune network in depression treatment. The holistic, multidrug, and multitarget nature of CHM represents an excellent example of systems medicine in the effective treatment of depression.
\end{abstract}

Keywords: Chinese herbal medicine; depression; neuroimmune system; neuroendocrine system; neuroendocrine-immune network; neuroinflammation; HPA axis

\section{Introduction}

Depression is a persistent and recurring mental illness, affecting more than 264 million people of all ages worldwide. It is also a major contributor to the global burden [1] and a leading cause of elevated disability [2]. Depression is clinically characterized by repeated depressive episodes, including anhedonia, insomnia, decreased speech, loss of interest and enjoyment, helplessness, and decreased energy [3]. Most researchers view depression as a multigenetic and multifactorial syndrome, which results from the complicated interplay of environmental and genetic factors and presents comorbidity with other diseases [4].

While current antidepressant medications, such as selective monoamine reuptake inhibitors and glutamate transmission-enhanced fast-acting antidepressants, can improve mental states of depression, these drugs are far from ideal, because they have severe side effects and low rates of efficacy [5]. Growing evidence suggests that central nervous system (CNS)-targeted medications alone are insufficient, and the development of novel medications or approaches for effective and systematic depression treatment is a pressing task $[4,6]$. In recent decades, many divergent biological systems have been identified to 
be involved in the pathogenesis of depression. In particular, studies have shown that the activation of the neuroinflammatory response of the immune system and hyperactivity of the hypothalamus-pituitary-adrenal (HPA) axis of the neuroendocrine system are two critical triggers in the etiology of depression [7] (Figure 1). It should be emphasized that communication or crosstalk exists between the neuroimmune and neuroendocrine systems and that the neuroendocrine-immune network plays a vital role in the systems biology of depression $[8,9]$.

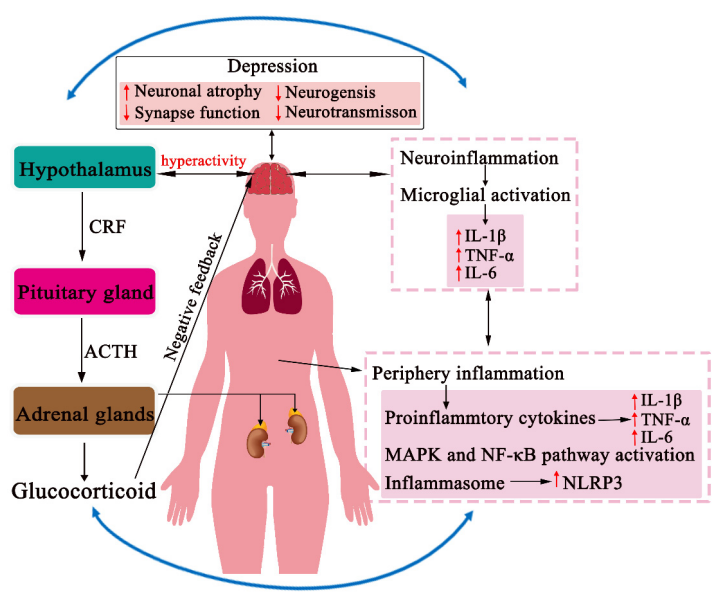

Figure 1. The neuroendocrine-immune network in the pathogenesis of depression. In response to stress, the peripheral or neuroimmune system activates the release of HPA axis hormones, whereas the stress-induced hyperactivation of the HPA axis also stimulates a proinflammatory or neuroinflammatory response. Intersystem crosstalk occurs at many levels through neural, immunological, and humoral interactions and subsequently results in the dysfunction of the central nervous system (CNS) in the pathogenesis of depression.

Preclinical studies have revealed that hyperactivity of the HPA axis can lead to the activation of the neuroinflammatory response of the immune system, whereas neuroinflammation can also modulate the activity of the HPA axis through various underlying mechanisms [8]. These findings have provided many novel pharmacological targets in either the neuroimmune or neuroendocrine system for depression treatment; however, none of these attempts have succeed in developing new medications directed toward these systems. Because of the intersystem crosstalk, agents that target one system alone will not be effective, and an additional medication that directly acts on the other system is also required to achieve a better treatment. Therefore, an improved approach to achieve an effective depression treatment should be systems biology-orientated and simultaneously target several biological systems involved in the pathogenesis of depression.

Traditional Chinese Medicine (TCM) is a holistic medicine that has been developed in China for centuries. It emphasizes the integration of a variety of biological systems in the human body and aims to prevent or heal diseases by maintaining or restoring internal homeostasis [4]. In TCM practice, a combination of multiple herbal drugs, socalled Chinese Herbal Medicine (CHM), is often used to act on multiple pharmacological targets simultaneously $[10,11]$. The systems biology-based, multi-target, and multi-drug medication is particularly suitable for the treatment of multigenetic and multifactorial diseases, such as depression [12].

Numerous CHM formulas are currently used for depression treatment in TCM practice $[11,13]$. Clinical studies have shown that these CHM antidepressant formulas exert comparable efficacies to conventional antidepressants, but with few adverse effects [14]. In addition, preclinical studies have demonstrated that CHM antidepressant formulas exhibit antidepressant-like activities in rodent models through multiple underlying mechanisms, and the de-hyperactivation of the HPA axis and anti-inflammation are the most common actions [15-17]. During the past decade, preclinical studies have extensively been 
performed by employing the molecular or systems pharmacology approach to uncover the mechanisms of action of CHM antidepressant formulas at both the molecular and systems levels. These studies have not only remarkably improved our understanding of the molecular basis and system-wide actions of CHM antidepressant formulas, but also promoted the development of novel medications for the effective and systematic treatment of depression [4].

In this narrative review, we aim to conclusively uncover the mechanism of action of CHM, specifically through the modulation of the neuroendocrine-immune network, by discussing the recent preclinical studies conducted using depressive animal models. The most recent literature showing that $\mathrm{CHM}$ constituents or formulas exert antidepressant activity by modulating the neuroinflammatory response of the immune system or the release of HPA axis hormones were prioritized in the selection of studies for discussion. According to their mechanisms of action, some representative CHM antidepressant constituents and formulas are summarized in each section, respectively. In addition, we also discuss the effects of CHM constituents and formulas on the neuroendocrine-immune network and the systems pharmacology approach in order to improve our understanding of the system-wide mechanisms of action of CHM formulas.

\section{Inflammation in the Pathogenesis of Depression}

Smith first proposed that inflammation may play a crucial role in the pathogenesis of depression in 1991 [18]. Since then, the immune system has been extensively studied to explore the mechanism by which the dysfunction of immune system is associated with symptoms of depression. Accumulating evidence has demonstrated that the dysregulation of the peripheral or neuroimmune system contributes to the pathogenesis of depression [19] (Figure 1). Clinical studies have indicated that patients suffering from depression showed significantly higher levels of proinflammatory cytokines, including interleukin-1 $\beta$ (IL-1 $\beta$ ), interleukin-6 (IL-6), tumor necrosis factor alpha (TNF- $\alpha$ ), C-reactive protein (CRP), and inflammasome, than healthy people [6,20-24]. These studies have also shown that patients with chronic peripheral inflammatory diseases have a higher incidence of depression [25]. In addition, over $50 \%$ of patients suffering from viral infections showed a depressive symptomatology after treatment with cytokine interferon-alpha (INF- $\alpha$ ) [26]. The reciprocal effects were also observed in rodent models. For instance, several studies have reported that chronic unpredictable mild stress (CUMS) treatment elevated the proinflammatory or neuroinflammatory response of the immune system in the blood and brain [27-29], whereas the administration of endotoxins, such as lipopolysaccharide (LPS), caused depressive-like behaviors by activating the indoleamine 2,3-dioxygenase (IDO) pathway [30] or proinflammatory cytokines [31]. These studies indicate that bidirectional communication exists between proinflammation or neuroinflammation and the CNS.

It is worth noting that the dysregulation of the peripheral immune system plays an important role in the pathogenesis of depression. Peripheral cytokines can be actively transported into the CNS through an increase in blood brain barrier (BBB) permeability $[19,32,33]$ and, subsequently, a reduction of serotonin neurotransmission and activation of the HPA axis [34]. Interestingly, low levels of proinflammatory cytokines regulate PI3K-Akt signaling to support synaptic function; however, abnormally increased proinflammatory cytokines contribute to damage, atrophy, and loss of spinal synapses through the modulation of signaling factors p38 and nuclear factor kappa B (NF-kB) [35].

Preclinical and clinical studies have also demonstrated that stress and depression are associated with an alteration in the morphology and activation of microglial cells, which leads to neuroinflammation and neuronal dysfunction [19,36]. In post-mortem histological and neuroimaging studies on depressive patients, robust changes in the microglial morphology and density in the prefrontal cortex (PFC) and hippocampus have been found $[19,37,38]$. In addition, a peritoneum injection of LPS into chronic neurodegenerative mice has been shown to result in a dramatic change in the microglia phenotype, which can transform into a proinflammatory phenotype through the overexpression of 
proinflammatory cytokines, such as IL-1 $\beta$, IL-6, and TNF- $\alpha[39,40]$. Furthermore, the activation of microglia mediates depressive-like behaviors through the shaping of the dendritic architecture and synaptic connection [41,42]. Taken together, these findings have provided evidence that the dysfunction of the neuroimmune system is involved in the pathogenesis of depression.

\section{CHM Regulation of the Neuroimmune System}

Many CHM herbal constituents exert anti-inflammation activity through various underlying mechanisms of action by regulating either proinflammatory cytokines, inflammatory signaling pathways or inflammasome [13,43-46]. While these studies have not revealed the specific drug-target interactions of these herbal constituents with their acting proteins, they have provided the molecular basis for understanding the mechanisms of action by which CHM herbs or formulas exert antidepressant activity through the modulation of the neuroimmune system. Figure 2 shows several representative CHM constituents that have been reported to exhibit antidepressant-like activity in depressive animal models, specifically by mediating the neuroimmune system. Other herbal constituents that possess anti-inflammation activity are also shown in Table 1. Some CHM formulas that produce antidepressant effects through the modulation of the neuroimmune system are listed in Table 2. In this section, we discuss the effects of CHM on the neuroimmune system according to its diverse pharmacological actions.
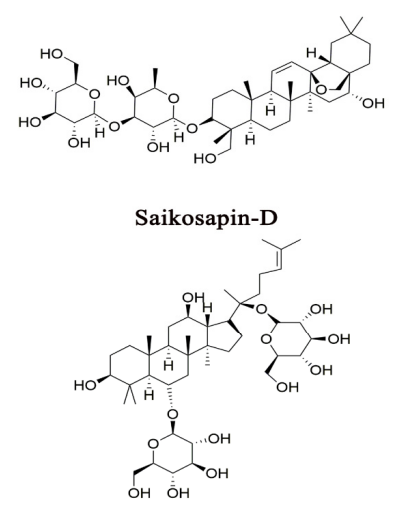

Ginsenoside Rg1

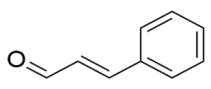

trans-cinnamaldehyde

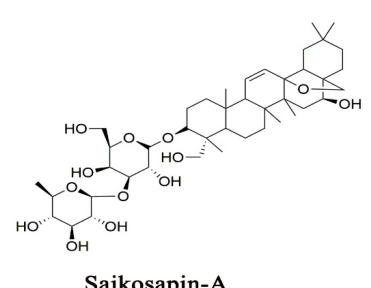

Saikosapin-A
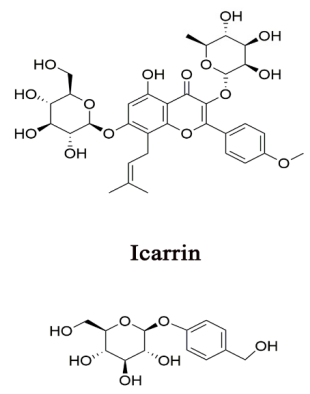

Gastrodin

Figure 2. Several representative CHM constituents that have been demonstrated to exert antidepressant activity, specifically by mediating the neuroimmune system.

\subsection{Proinflammatory Cytokines and Cytokine Receptors}

Proinflammatory cytokines are mainly derived from immune cells such as monocytes, macrophages, lymphocytes, and dendritic cells, acting as important modulators of neuroinflammatory response [6]. Recently, several studies have investigated the influence of peripheral proinflammatory cytokines (e.g., IL-6, IL-1 $\beta$, and TNF- $\alpha$ ) on neuronal synaptic plasticity, neurogenesis, and neuromodulation, which play critical roles in the initiation, relapse, and progression of depression $[47,48]$. It is worth noting that these proinflammatory cytokines are usually maintained at low levels under physiological conditions; however, its levels are increased by approximately 100-fold under stress-related pathological conditions [6]. In addition, microglia can release proinflammatory cytokines that influence the neurobiology of depression by decreasing the number and function of astrocytes [19]. 
Furthermore, cytokine receptors have also been demonstrated to produce neurobiological effects on microglia activation and neuroinflammation [19].

Bupleurum chinense DC has commonly been used to treat inflammation and infectious diseases [49]. Saikosaponin-D, a triterpenoid saponin isolated from Bupleurum chinense DC, has multiple pharmacological effects, such as anti-inflammation [50] and antidepressant activity [51]. A study has shown that pretreatment with saikosaponin-D (1 mg/kg, 7 days, i.g.) inhibited LPS-induced microglia activation and suppressed the secretion of proinflammatory cytokines (IL-1 $\beta$, IL-6, and TNF- $\alpha$ ) [52]. Its mechanism of action has been suggested to inhibit the transportation of high mobility group box 1 (HMGB1), a late inflammatory factor, into extracellular space, which results in the downregulation of the Toll-like receptors 4 (TLR4)/NF- $\mathrm{kB}$ pathway in both the hippocampus in mice and primary microglia cells. Saikosaponin-A (50 or $100 \mathrm{mg} / \mathrm{kg}$, 4 weeks, p.o.), a derivative of saikosaponin-D, has also been demonstrated to exhibit antidepressant-like activity through its effects on the neuroimmune system by suppressing the CUMS-induced IL- $1 \beta$, IL- 6 , and TNF- $\alpha$ overexpression in the hippocampus in rats [53]. Taken together, these results indicate that triterpenoid saponins exert antidepressant-like activities in animal models by reducing proinflammatory cytokine levels; however, further study is needed to explore their molecular interactions with the potential acting proteins and their pharmacological profiles.

While no CHM constituents have been reported to exert a specific action on cytokine receptors, a CHM formula comprised of eight herbs for the treatment of postpartum depression, known as the Shen-Qi-Jie-Yu decoction $(1.25 \mathrm{~g} / \mathrm{mL}, 1,2,4$ weeks, i.g.), has recently been shown to produce antidepressant effects by decreasing the expression of cytokine receptors, such as IL- $1 \mathrm{R}_{1}$ and glycoprotein 130 , in the hippocampus in a rat model of postpartum depression [54]. However, this study did not clarify the molecular mechanism by which the herbal formula produces profound effects on the expression of cytokine receptors.

\subsection{Proinflammatory Signaling Pathway}

Increasing evidence has suggested that proinflammatory signaling pathways, such as the mitogen-activated protein kinase (MAPK) pathway and NF- $\mathrm{kB}$ pathway, influence BBB integration, microglia activation, and neurogenesis [6,55]. A bioinformatic analysis has identified that the MAPK pathway is one of the functionally enriched signaling pathways in the neurobiology of depression [56]. Extracellular regulated kinases (ERK1/2), c-Jun N-terminal kinases (JNKs), and p38 MAP kinases are three subfamilies of the MAPK signaling pathway. It has been demonstrated that the MAPK pathway is involved in the differentiation of astrocytes and other neuronal cells, synaptic plasticity, and neuron survival, as evidenced by the robust changes of MAPK signaling in the hippocampus in depressive animal models [6,57-59]. These kinase subfamilies play central roles in the release of proinflammatory cytokines or the activation of NF- $\mathrm{KB}$, a proinflammatory transcription factor, in response to various stimuli, such as psycho-emotional stressors, acute alcohol exposure, pathogenic products, or proinflammatory cytokines [60-62]. NF- $\mathrm{kB}$ is abundantly distributed in the brain, microglia, BBB, and peripheral immune responsive cells $[60,61,63]$, and it is an essential mediator in several important physiological processes, including synaptogenesis, neurotransmission, neuroprotection, and neuroinflammation [64-66]. In animal models, NF- $\mathrm{kB}$ activity can be elevated by the degradation of NF- $\mathrm{\kappa B}$ kinase inhibitors [67], and it can also be activated by IL- $1 \beta$ signaling and proinflammatory cytokines released from both the peripheral immune cells and the brain $[68,69]$. A study showed that proinflammatory cytokine overexpression and behavioral abnormality can be reduced by the blockade of NF- $\mathrm{B}$ signaling [68]. Hence, these findings highlight the critical roles of the MAPK and NF- $\mathrm{KB}$ signaling pathways in the pathogenesis of depression.

Many $\mathrm{CHM}$ herbal constituents have been shown to exert antidepressant-like activities in animal models by inhibiting the MAPK or NF- $\mathrm{KB}$ signaling pathway [70,71]. Ginsenoside Rg1, a saponin extracted from Panax ginseng C. A. Mey., has been demonstrated to act as 
a potential neuroprotective agent in depression treatment [72,73]. The administration of ginsenoside $\operatorname{Rg} 1$ ( 20 or $40 \mathrm{mg} / \mathrm{kg}$, 34 days, i.g.) inhibited hippocampal neuroinflammation and reduced the expression of proinflammatory cytokines and microglial activation through the inhibition of NF- $\mathrm{kB}$ activity by the MAPK and silent information regulator 2 homolog 1 signaling pathways in chronic social defeat stress mice [72]. Additionally, ginsenoside $\mathrm{Rg} 1$ (10 or $20 \mathrm{mg} / \mathrm{kg}$, 3 days, p.o.) has also been revealed to significantly reduce the $\mathrm{p}-\mathrm{I} \kappa \mathrm{B}$ level in cytoplasm and nuclear translocation of NF- $\mathrm{kB}$ in LPS-induced depressive mice, probably through the suppression of iNOS and TNF- $\alpha$ production in the brain [74]. Moreover, in comparison with the LPS-induced model, the levels of the MAPK subfamily, such as p38 MAPK, ERK, and JNK, were reversed through treatment with Rg1 [74]. These studies have indicated that the inhibitory effects of Rg1 are meditated by inhibiting the NF- $\mathrm{KB}$ and MAPK pathways. While these findings have provided novel insights into the therapeutic implications of ginsenoside Rg1 for depression treatment, further study is needed to determine if the herbal constituent directly interacts with the neuroinflammatory signaling pathways and what pharmacological targets it specifically acts on.

\subsection{Inflammasome}

Inflammasome, a complex of multiple proteins, functions as an intracellular sensor in response to environmental and cellular stress [75-77]. In particular, the NLRP3 inflammasome complex, the well-studied inflammasome member, acts as a key convergent molecular pathway in several mechanisms of peripheral and central inflammatory responses in neurological and inflammatory diseases [78]. NLRP3 inflammasome can be released by microglia, macrophages, and astrocytes in the CNS [77]. NLRP3 activation, induced by the stimulation of either Toll-like receptors (TLRs) or adenosine triphosphate (ATP), results in caspase- 1 activation and IL- $1 \beta$ maturation, which initiate inflammatory responses [77]. Clinical studies have demonstrated that the gene expression of NLRP3, caspase-1, and IL-1 $\beta$ are elevated in blood samples of depressive patients [79]. In addition, it has also been shown that, in rodent models, the NLRP3 inflammasome is significantly elevated in depressive brain tissues and that the administration of an NLRP3 inhibitor improves depressive-like behaviors induced by LPS or CUMS [80,81]. Furthermore, Su et al. has suggested that NLRP3 inflammasome modulates depressive-like behaviors through the regulating activities of the MAPK and NF- $\mathrm{KB}$ pathways [55]. Taken together, these studies indicate that the activation of NLRP3 inflammasome signaling is involved in the pathogenesis of depression and that inflammasome serves as a potential pharmacological target in depression treatment.

Studies have shed light on depression treatments involving CHM constituents that suppress the overexpression or activation of NLRP3. For example, trans-cinnamaldehyde $(10 \mu \mathrm{M}, 24 \mathrm{~h})$, a bioactive constituent of Cinnamomum cassia Presl, has been shown to inhibit microglia activation and, subsequently, to alleviate inflammatory responses to various stressors [82]. The administration of trans-cinnamaldehyde ( $10 \mathrm{mg} / \mathrm{kg}, 3$ weeks, p.o.) has been shown to increase sucrose preference and reduce the immobility time in CUMS rats. The study has also shown that trans-cinnamaldehyde attenuated the expression of NF- $\mathrm{kB}$ pathway components, including TLR-4, I $\kappa$ B $\alpha, \mathrm{p} 65$, NF- $\kappa \mathrm{B}-1$, and TNF- $\alpha$, and downregulated the expression of NLRP3, caspase-1, IL-1 $\beta$, and IL-18. These observations suggest that trans-cinnamaldehyde produces antidepressant effects through the inactivation of the NF- $\mathrm{KB} / \mathrm{NLRP3}$ inflammasome pathway in animal models [28]. Moreover, a recent study has suggested that icariin ( 20 or $40 \mathrm{mg} / \mathrm{kg}$, 35 days, p.o.), a prenylated flavonoid extracted from Epimedium brevicornu Maxim, exerts anti-inflammation effects and ameliorates oxidative stress-induced brain damage by inactivating the NF- $\kappa \mathrm{B}$ signaling and inhibiting the NLRP3-inflammasome/caspase-1/IL-1 $\beta$ axis in the hippocampus [83]. It would be interesting to identify their specific targeting proteins in the inflammasome complex, which would, in turn, improve our understanding of the mechanisms of action by which $\mathrm{CHM}$ constituents modulate the neuroimmune system by specifically interacting with inflammasomes. 
Table 1. Constituents of CHM that modulate the release of HPA axis hormones and exhibit anti-inflammatory and antidepressant-like activities in animal models of depression.

\begin{tabular}{|c|c|c|c|c|c|c|c|}
\hline Herb & Herbal Constituent & Animal Model & Behavioral Test & $\begin{array}{l}\text { Administration } \\
\text { Dose/Time/Route of Treatment }\end{array}$ & $\begin{array}{l}\text { Effects on Mediators of } \\
\text { Inflammation }\end{array}$ & $\begin{array}{l}\text { Effects on } \\
\text { Hormones of the } \\
\text { HPA Axis }\end{array}$ & Reference \\
\hline Fallopia multiflora (Thunb.) Harald. & $\begin{array}{l}\text { 2,3,5, 4'-Tetrahydroxystilbene- } \\
\text { 2-O- } \beta \text {-D-glucoside }\end{array}$ & $\begin{array}{l}\text { LPS-induced } \\
\text { depressive mice }\end{array}$ & TPT, FST, SPT & $30,60 \mathrm{mg} / \mathrm{kg}, 7$ days, i.p. & $\begin{array}{l}\downarrow \text { IL- } 6, \text { TNF- } \alpha, \text { IL-1 } 1 \beta \text { in } \\
\text { hippocampus and PFC }\end{array}$ & ND & [84] \\
\hline Polygala tenuifolia Willd. & Senegenin & CUMS mice & TST, FST, SPT & $4,8 \mathrm{mg} / \mathrm{kg}, 21$ days, i.g. & $\begin{array}{l}\downarrow N F-\kappa B / N L R P 3 \text { signal pathway } \\
\text { in hippocampus }\end{array}$ & ND & [86] \\
\hline Gastrodia elata Bl. & Gastrodin & CUS rats & SPT, FST, Morris water test & $50,100,200 \mathrm{mg} / \mathrm{kg}, 14$ days, i.p. & $\begin{array}{l}\downarrow \text { NF- } \kappa B \text { and IL- } 1 \beta \text { expression } \\
\text { in hippocampus }\end{array}$ & ND & [87] \\
\hline Cinnamomum cassia Presl & Trans-Cinnamaldehyde & CUMS rats & Sucrose consumptions, FST & $10 \mathrm{mg} / \mathrm{kg}$, 3 weeks, p.o. & $\begin{array}{l}\downarrow \text { IL-1 } \beta, \text { IL- } 18, \text { TNF- } \alpha \text { in serum } \\
\downarrow \text { NF- } k \text { B } / N L R P 3 \text { in PFC } \\
\text { and hippocampus }\end{array}$ & ND & [28] \\
\hline Lonicera japonica Thunb. & $\begin{array}{l}\text { Lonicera } \\
\text { japonicapolysaccharide }\end{array}$ & CUMS mice & OFT, EPM, TST, FST & $30,100 \mathrm{mg} / \mathrm{kg}, 21$ days, i.g. & $\begin{array}{l}\downarrow \text { NLRP3, IL-1 } \beta \text {, caspase-1 } \\
\text { in hippocampus }\end{array}$ & ND & [88] \\
\hline Andrographis paniculata (Burm. f.) Nees & Andrographolide & CUMS mice & FST, SPT, TST, Y maze & $2.5,5 \mathrm{mg} / \mathrm{kg}, 14$ days, p.o. & $\begin{array}{l}\downarrow \text { IL-1 } \beta, \text { IL-6, TNF- } \alpha, \text { NF- } \kappa B \\
\text { signaling, NLRP3 in PFC }\end{array}$ & ND & [89] \\
\hline $\begin{array}{l}\text { Houpoea officinalis (Rehder and E. H. } \\
\text { Wilson) N. H. Xia and C. Y. Wu }\end{array}$ & Honokiol & $\begin{array}{l}\text { LPS-induced } \\
\text { depressive mice }\end{array}$ & FST, TST, & $2.5,510 \mathrm{mg} / \mathrm{kg}$, 11 days, p.o. & $\begin{array}{l}\downarrow \text { NF- } \kappa \text { B activation } \\
\text { in hippocampus } \\
\downarrow \text { IL- } 1 \beta, \text { TNF- } \alpha, \text { IFN- } \gamma \text { in serum }\end{array}$ & ND & {$[90]$} \\
\hline \multirow{3}{*}{ Perilla frutescens (Linn.) Britt. } & \multirow{2}{*}{ Perilla aldehyde } & CUMS rats & SPT, FST, OFT & $20,40 \mathrm{mg} / \mathrm{kg}$, 3 weeks, i.g. & $\begin{array}{l}\downarrow \text { TNF- } \alpha, \text { IL- } 1 \beta \text { in hippocampus } \\
\downarrow \text { NLRP3 in hippocampus }\end{array}$ & ND & [92] \\
\hline & & $\begin{array}{l}\text { LPS-induced } \\
\text { depressive mice }\end{array}$ & TST, FST & $60,120 \mathrm{mg} / \mathrm{kg}, 7$ days, i.g. & $\downarrow$ TNF- $\alpha$, IL-6 in serum and PFC & ND & [93] \\
\hline & Essential oil of Perilla frutescens & CUMS mice & OFT, TST, FST, SPT & 3, 6, $9 \mathrm{mg} / \mathrm{kg}, 3$ weeks, g.i. & $\downarrow$ TNF- $\alpha$, IL-6, IL-1 in plasma & ND & [94] \\
\hline Polygonum aviculare $\mathrm{L}$. & Polygonum aviculare L. extract & $\begin{array}{l}\text { Restraint-stressed } \\
\text { mice }\end{array}$ & FST, SPT, OFT & 100, $200 \mathrm{mg} / \mathrm{kg}, 15$ days, p.o. & $\downarrow$ TNF- $\alpha$, IL- 6, IL- $1 \beta$ in the brain & ND & [95] \\
\hline Hemerocallis fulva $(\mathrm{L}.) \mathrm{L}$. & Ethanol extracts & $\begin{array}{l}\text { LPS-induced } \\
\text { depressive mice }\end{array}$ & SPT & 180 mg $/ \mathrm{kg}, 7$ days, p.o. & $\begin{array}{l}\downarrow \text { NF-kB signaling pathway } \\
\text { in PFC }\end{array}$ & ND & [96] \\
\hline Angelica sinensis (Oliv.) Diels & Ferulic Acid & CUMS mice & SPT & $20,40,80 \mathrm{mg} / \mathrm{kg}$, 4 weeks, p.o. & $\begin{array}{l}\downarrow \text { TNF- } \alpha, \text { IL-6, IL-1 } \beta \text {, microglial } \\
\text { activation, NF- } \mathrm{KB} \text { and NLRP3 } \\
\text { in PFC }\end{array}$ & ND & [97] \\
\hline Paeonialactiflora Pall & Paeoniflorin & $\begin{array}{l}\text { IFN- } \alpha \text {-induced } \\
\text { depressive mice }\end{array}$ & SPT, OFT, TST, FST & $10,20,40 \mathrm{mg} / \mathrm{kg}, 4$ weeks, i.g. & $\begin{array}{l}\downarrow \text { TNF- } \alpha \text {, IL-6, IL-1 } \beta, \text { IL-9, IL-10, } \\
\text { IL-12, MCP-1 in serum, mPFC, } \\
\text { vHi and amygdala }\end{array}$ & ND & [98] \\
\hline
\end{tabular}


Table 1. Cont

\begin{tabular}{|c|c|c|c|c|c|c|c|}
\hline Herb & Herbal Constituent & Animal Model & Behavioral Test & $\begin{array}{l}\text { Administration } \\
\text { Dose/Time/Route of Treatment }\end{array}$ & $\begin{array}{l}\text { Effects on Mediators of } \\
\text { Inflammation }\end{array}$ & $\begin{array}{l}\text { Effects on } \\
\text { Hormones of the } \\
\text { HPA Axis }\end{array}$ & Reference \\
\hline Xiaobuxin-Tang & Total flavonoid extract & $\begin{array}{l}\text { LPS-induced } \\
\text { depressive mice }\end{array}$ & TST, FST & $25,50,100 \mathrm{mg} / \mathrm{kg}, 1 \mathrm{~h}$, i.p. & $\downarrow$ TNF- $\alpha$, IL- $1 \beta$ in the barin & ND & [99] \\
\hline Ginkgo biloba $\mathrm{L}$. & EGb761 & $\begin{array}{l}\text { LPS-induced } \\
\text { depressive mice }\end{array}$ & FST, TST, SPT & $50,100,150 \mathrm{mg} / \mathrm{kg}, 10$ days, p.o. & $\begin{array}{l}\downarrow \text { IL-6, TNF- } \alpha \text {, IL-1 } \beta, \text { IL-17A } \\
\text { in hippocampus } \\
\uparrow \text { IL-10 in hippocampus }\end{array}$ & ND & {$[100]$} \\
\hline Pueraria lobate (Willd.) Ohwi & Puerarin & CUS rats & SPT, NSFT, FST & $30,60,120 \mathrm{mg} / \mathrm{kg}, 20$ days, i.g. & ND & $\begin{array}{l}\downarrow \mathrm{CRH}, \mathrm{CORT}, \\
\mathrm{ACTH} \text { in serum }\end{array}$ & [101] \\
\hline Tribulus terrestris Linnaeus & Tribulus Terrestris Saponins & CMS rats & OFT, SPT & $0.375,0.75,2.25 \mathrm{~g} / \mathrm{kg}, 4$ weeks, i.g. & ND & $\begin{array}{l}\downarrow \mathrm{CRH}, \mathrm{CORT} \\
\text { in serum }\end{array}$ & [102] \\
\hline $\begin{array}{l}\text { Rehmannia glutinosa (Gaert.) Libosch. } \\
\text { ex Fisch. et Mey. }\end{array}$ & Ethanol extracts & CUMS rats & SPT & $150,300,600 \mathrm{mg} / \mathrm{kg}, 3$ weeks, p.o. & ND & $\downarrow$ CORT in serum & [103] \\
\hline \multirow{3}{*}{ Panax ginseng C.A. Meyer } & Ginseng total saponins & $\begin{array}{l}\text { LPS-induced } \\
\text { depressive } \\
\text { mice/RAW264.7 cells; } \\
\text { CUMS rats }\end{array}$ & FST, TST, SPT & $\begin{array}{l}200 \mathrm{mg} / \mathrm{kg}, 7 \text { days, i.g.; } 12.5,25, \\
50 \mathrm{mg} / \mathrm{kg}, 6 \text { weeks, i.g. }\end{array}$ & $\begin{array}{l}\downarrow \text { IL- } 1 \beta, \text { IL- } 6 \text {, TNF- } \alpha, \text { IDO mRNA } \\
\text { in hippocampus }\end{array}$ & $\begin{array}{l}\downarrow \text { CORT in serum } \\
\uparrow \text { GR mRNA } \\
\text { in hippocampus }\end{array}$ & {$[104,105]$} \\
\hline & Ginsenoside Rg1 & $\begin{array}{l}\text { CSDS mice; } \\
\text { CUMS rats }\end{array}$ & $\begin{array}{l}\text { Social interaction test, SPT, } \\
\text { FST, TST }\end{array}$ & $\begin{array}{l}20,40 \mathrm{mg} / \mathrm{kg}, 34 \text { days, i.g.; } 5,10, \\
20 \mathrm{mg} / \mathrm{kg}, 28 \text { days, i.g. }\end{array}$ & $\begin{array}{l}\downarrow \mathrm{IL}-6, \mathrm{TNF}-\alpha, \text { IL-1 } \beta, \text { microglial } \\
\text { activation, p-NF- } \mathrm{kB} \\
\text { in hippocampus }\end{array}$ & $\begin{array}{l}\downarrow \text { CORT level } \\
\text { in serum } \\
\uparrow \text { GR protein in } \\
\text { PFC and hip- } \\
\text { pocampus }\end{array}$ & {$[72,106]$} \\
\hline & Ginsenoside Rg3 & $\begin{array}{l}\text { LPS-induced } \\
\text { depressive mice; } \\
\text { CUS rats }\end{array}$ & $\begin{array}{l}\text { TPT, FST, EPMT, NSFT, } \\
\text { OFT }\end{array}$ & $\begin{array}{l}20,40 \mathrm{mg} / \mathrm{kg}, 3 \text { days, i.g.; } 10,20, \\
40 \mathrm{mg} / \mathrm{kg}, 14 \text { days, i.g. }\end{array}$ & $\begin{array}{l}\downarrow \text { IL-6, TNF- } \alpha \text { in plasma } \\
\downarrow \text { IL-6, IL- } 1 \beta, \text { IDO, microglial } \\
\text { activation, NF- } \kappa \text { B pathway in } \\
\text { brain }\end{array}$ & $\begin{array}{l}\downarrow \mathrm{CRH}, \mathrm{CORT}, \\
\mathrm{ACTH} \text { in serum }\end{array}$ & {$[107,108]$} \\
\hline Salvia miltiorrhiza Bunge & Salvianolic acid B & CMS mice & SPT, FST, TST & $20 \mathrm{mg} / \mathrm{kg}$, 3 weeks, i.p. & $\begin{array}{l}\downarrow \text { IL- } 1 \beta, \text { TNF- } \alpha \text { in hippocampus } \\
\text { and cortex } \\
\uparrow \text { IL- } 10 \text {, TGF- } \beta \text { in hippocampus } \\
\text { and cortex }\end{array}$ & $\begin{array}{l}\downarrow \text { CORT } \\
\text { in plasma }\end{array}$ & [109] \\
\hline Aquilaria spp. & $\begin{array}{l}\text { Agarwood } \\
\text { Essential Oil }\end{array}$ & $\begin{array}{l}\text { Restraint } \\
\text { stress-induced mice }\end{array}$ & TST, FST & $10,20,40 \mathrm{mg} / \mathrm{kg}, 10$ days, i.p. & $\downarrow$ IL-1 $\beta$, IL- $1 \alpha$, IL-6 in serum & $\begin{array}{l}\downarrow \text { CRF, CRF } \\
\text { receptor in cortex } \\
\downarrow \text { CORT, ACTH } \\
\text { in serum }\end{array}$ & [110] \\
\hline Chaihu-Shugan-San & Saikosaponin A & CUMS rats & SPT, NPFT, FST & 25,50 or $100 \mathrm{mg} / \mathrm{kg}$, 4 weeks, p.o. & $\begin{array}{l}\downarrow \text { IL- } 1 \beta, \text { IL-6, TNF- } \alpha \\
\text { in hippocampus }\end{array}$ & $\begin{array}{l}\downarrow \mathrm{CRH} \text { in } \\
\text { hypothalamus } \\
\downarrow \text { GR mRNA } \\
\text { in hippocampus }\end{array}$ & {$[53]$} \\
\hline Rhodiola rosea $\mathrm{L}$. & Salidroside & OBX rats & TST, FST, SPT & $\begin{array}{l}20,40 \mathrm{mg} / \mathrm{kg}, 2 \text { weeks, p.o.; } 20, \\
40 \mathrm{mg} / \mathrm{kg} \text {, } 2 \text { weeks, i.g. }\end{array}$ & $\begin{array}{l}\downarrow \text { TNF- } \alpha \text {, IL- } 1 \beta \text { in hippocampus } \\
\downarrow \text { IL- } 1 \beta \text {, IL- } 6 \text {, TNF- } \alpha, \text { NF- } \mathrm{B} \\
\text { activation in PFC }\end{array}$ & $\begin{array}{l}\uparrow \text { GR in } \\
\text { hippocampus } \\
\downarrow \text { CRH in } \\
\text { hypothalamus }\end{array}$ & {$[111,112]$} \\
\hline
\end{tabular}


Table 1. Cont.

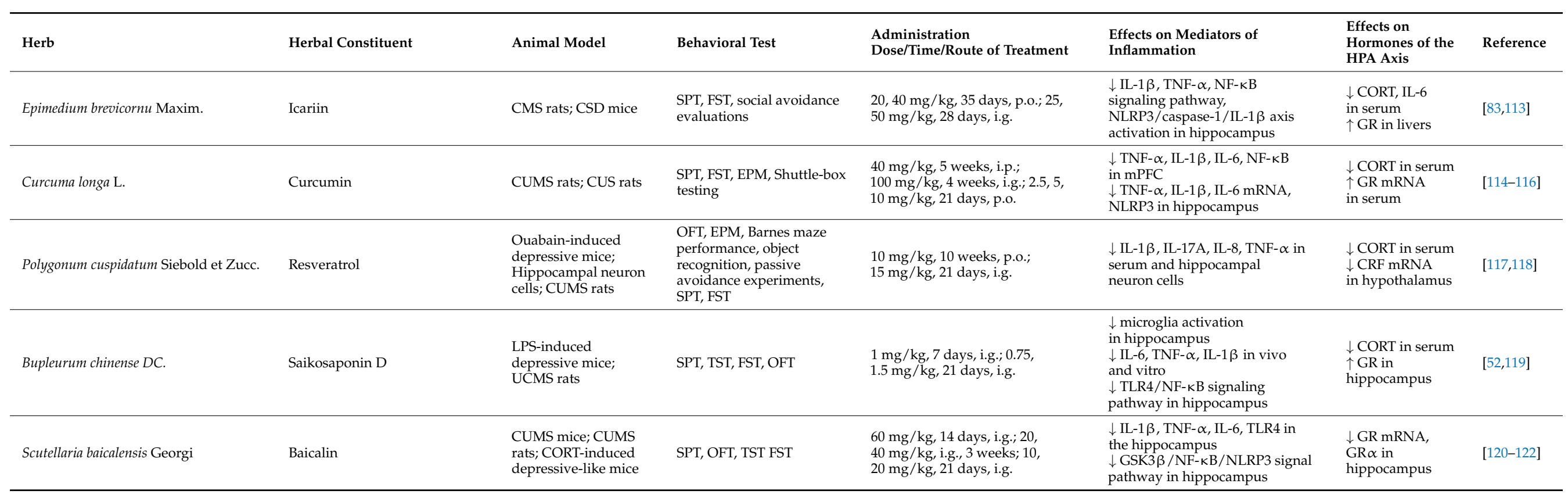


Table 2. CHM formulas traditionally used in TCM for the treatment of depression, which exhibit anti-inflammatory activity and modulate the release of HPA axis hormones.

\begin{tabular}{|c|c|c|c|c|c|c|c|c|}
\hline CHM Formula & $\begin{array}{l}\text { Plant Name/Ratio in Fixed } \\
\text { Combination }\end{array}$ & Daily Human Dose & Animal Model & Behavioral Test & $\begin{array}{l}\text { Administration } \\
\text { Dose/Time/Route of } \\
\text { Treatment }\end{array}$ & $\begin{array}{l}\text { Effects on } \\
\text { Mediators of } \\
\text { Inflammation }\end{array}$ & $\begin{array}{l}\text { Effects on } \\
\text { Hormones of } \\
\text { the HPA Axis }\end{array}$ & Reference \\
\hline Xiaoyao Pills & $\begin{array}{l}\text { Bupleurum chinense DC., } \\
\text { Osmanthus fragrans var. } \\
\text { aurantiacus Makino, Paeonia } \\
\text { lactiflora Pall., Smilax glabra Roxb, } \\
\text { Atractylodes macrocephala Koidz., } \\
\text { Mentha haplocalyx Briq., Zingiber } \\
\text { officinale Roscoe and Glycyrrhiza } \\
\text { uralensis Fisch.; 3:3:3:3:3:1:2:1.5 }\end{array}$ & 2 times/day & $\begin{array}{l}\text { LPS-induced } \\
\text { depressive mice/rats }\end{array}$ & TST, FST, OFT, NSFT & $\begin{array}{l}0.4836,0.93,1.86 \mathrm{~g} / \mathrm{kg} \text {, } \\
14 \text { days, i.g. }\end{array}$ & $\begin{array}{l}\downarrow \text { IL- } 6 \text { in serum } \\
\text { and hippocampus } \\
\downarrow \text { TNF- } \alpha \text { in } \\
\text { hippocampus and } \\
\text { cortex }\end{array}$ & ND & [123-125] \\
\hline $\begin{array}{l}\text { Mahuang-Fuzi-Xixin } \\
\text { Decoction }\end{array}$ & $\begin{array}{l}\text { Aconitum carmichaeli Pcbx., } \\
\text { Ephedra sinica Stapf and Asarum } \\
\text { sieboldii Miq.; 3:2:1 }\end{array}$ & 3 times/day & $\begin{array}{l}\text { LPS-induced } \\
\text { depressive mice }\end{array}$ & SPT, OFT, TST, FST & $\begin{array}{l}2.5,12.5,25 \mathrm{~g} / \mathrm{kg} \text {, } \\
1 \text { week, p.o. }\end{array}$ & $\begin{array}{l}\downarrow \text { IL-1 } \beta, \text { NLRP3 } \\
\text { in hippocampus }\end{array}$ & ND & [126] \\
\hline $\begin{array}{l}\text { Jieyu Anshen } \\
\text { granule }\end{array}$ & $\begin{array}{l}\text { Bupleurum abchasicum Manden., } \\
\text { Ziziphus jujuba Mill., Dens } \\
\text { Draconis, Polygala tenuifolia } \\
\text { Willd., Lilium brownie var. } \\
\text { viridulum Baker, Atractylodes } \\
\text { macrocephala Koidz., Triticum } \\
\text { aestivum L., Angelica sinensis } \\
\text { (Oliv.) Diels, Acorus tatarinowii } \\
\text { Schott, Pinellia ternate (Thunb.) } \\
\text { Makino, Glycyrrhiza uralensis } \\
\text { Fisch., Gardenia jasminoides J. Ellis, } \\
\text { Arisaema Cum Bile, Curcuma longa } \\
\text { L., Smilax glabra Roxb., and } \\
\text { Fructus Jujubae.; } \\
\text { 4:5:10:4:10:3:10:3:4:3:3:4:4:4:5:3 }\end{array}$ & $\begin{array}{l}5 \mathrm{~g} ; \\
2 \text { times/day }\end{array}$ & PSD rats & $\begin{array}{l}\text { OFT, SPT, water } \\
\text { maze test }\end{array}$ & $1,3 \mathrm{~g} / \mathrm{kg}, 4$ weeks, i.g. & $\begin{array}{l}\downarrow N F-k B \text { signaling in } \\
\text { PFC and } \\
\text { hippocampus }\end{array}$ & ND & {$[127,128]$} \\
\hline Jiaotai wan & $\begin{array}{l}\text { Coptis chinensis Franch. and } \\
\text { Cinnamomum cassia.; 10:1 }\end{array}$ & $1.5-2.5 \mathrm{~g} /$ day & $\begin{array}{l}\text { LPS-induced } \\
\text { depressive mice }\end{array}$ & TST, FST, SPT, OFT & $\begin{array}{l}4.2,8.4 \mathrm{~g} / \mathrm{kg}, \\
7 \text { days, i.g. }\end{array}$ & $\begin{array}{l}\downarrow \mathrm{TNF}-\alpha, \text { IL-6 } \\
\text { in serum } \\
\downarrow \text { NF- } \mathrm{B} \text { signaling } \\
\text { in brain }\end{array}$ & ND & {$[129,130]$} \\
\hline
\end{tabular}


Table 2. Cont

\begin{tabular}{|c|c|c|c|c|c|c|c|c|}
\hline CHM Formula & $\begin{array}{l}\text { Plant Name/Ratio in Fixed } \\
\text { Combination }\end{array}$ & Daily Human Dose & Animal Model & Behavioral Test & $\begin{array}{l}\text { Administration } \\
\text { Dose/Time/Route of } \\
\text { Treatment }\end{array}$ & $\begin{array}{l}\text { Effects on } \\
\text { Mediators of } \\
\text { Inflammation }\end{array}$ & $\begin{array}{l}\text { Effects on } \\
\text { Hormones of } \\
\text { the HPA Axis }\end{array}$ & Reference \\
\hline $\begin{array}{l}\text { Shen-Qi-Jie-Yu } \\
\text { Decoction }\end{array}$ & $\begin{array}{l}\text { Astragalus membranaceus (Fisch) } \\
\text { Bunge, Curcuma aromatica Salisb, } \\
\text { Ziziphus jujuba var spinosa } \\
\text { (Bunge) Hu ex HF Chow, Cornus } \\
\text { officinalis Sieb et Zucc } \\
\text { (Cornaceae), Codonopsis pilosula } \\
\text { (Franch) Nannf, Citrus reticulata } \\
\text { Blanco, Citrus medica L, and } \\
\text { Angelica sinensis (Oliv) Diels.; } \\
\text { 10:7.5:7.5:7.5:6:5:5:5 }\end{array}$ & 1 time/day & $\begin{array}{l}\text { Postpartum } \\
\text { depressive rat model }\end{array}$ & OFT, SPT, FST & $\begin{array}{l}1.25 \mathrm{~g} / \mathrm{mL}, 1,2, \\
4 \text { weeks, i.g. }\end{array}$ & $\begin{array}{l}\downarrow \text { IL-1 } \beta \text { and IL-6 } \\
\text { in serum } \\
\downarrow \text { IL-1RI and gp130 } \\
\text { in hippocampus }\end{array}$ & ND & [54] \\
\hline $\begin{array}{l}\text { Jieyuanshen } \\
\text { Decoction }\end{array}$ & $\begin{array}{l}\text { Bupleurum chinense DC., } \\
\text { Scutellaria baicalensis Georgi, } \\
\text { Ziziphusjujuba Mill. var. spinosa } \\
\text { (Bunge) Hu ex H.F. Chou, } \\
\text { Glycyrrhiza uralensis Fisch., Lilium } \\
\text { brownie F.E. Brown var. viridulum } \\
\text { Baker, and Pinelliaternata (Thunb.) } \\
\text { Breit.; 1:1.5:0.5:1:1:3 }\end{array}$ & 2 times/day & CUS rats & SPT, OFT & $\begin{array}{l}\text { 8.2, } 16.3,32.7 \mathrm{~g} / \mathrm{kg} \text {, } \\
28 \text { days, i.g. }\end{array}$ & ND & $\begin{array}{l}\downarrow \mathrm{CORT}, \mathrm{ACTH}, \\
\text { CRH in serum } \\
\uparrow \mathrm{GR} \text { in } \\
\text { hippocampus }\end{array}$ & [131] \\
\hline Shuyu San & $\begin{array}{l}\text { Bupleurum chinense DC., Curcuma } \\
\text { aromatica Salisb., Mentha } \\
\text { canadensis Linnaeus, Gardenia } \\
\text { jasminoides Ellis, Smilax glabra } \\
\text { Roxb., Polygala tenuifolia Willd., } \\
\text { Acorus gramineus Soland., } \\
\text { Ziziphus jujuba var. spinosa } \\
\text { (Bunge) Hu ex H. F. Chow., and } \\
\text { Albizia julibrissin Durazz.; } \\
\text { 5:7.5:3:5:5:5:5:7.5:5 }\end{array}$ & 2 times/day & UCMS rats & TST, FST & $\begin{array}{l}2.5,7.5,25 \mathrm{~g} / \mathrm{kg} \text {, } \\
3 \text { weeks, g.p. }\end{array}$ & ND & $\begin{array}{l}\downarrow \mathrm{CRH}, \mathrm{ACTH}, \\
\text { CORT in serum }\end{array}$ & [133] \\
\hline Chaihu-Shugan-San & $\begin{array}{l}\text { Bupleurum chinense DC., Citrus } \\
\text { reticulata Blanco, Ligusticum } \\
\text { sinense 'Chuanxiong', Cyperus } \\
\text { rotundus L., Citrus } \times \text { aurantium } \\
\text { Linnaeus, Paeonia lactiflora Pall., } \\
\text { and Glycyrrhiza uralensis Fisch.; } \\
\text { 4:4:3:3:3:3:1 }\end{array}$ & 2 times/day & $\begin{array}{l}\mathrm{ApoE}^{-/-} \text {mice; } \mathrm{UMS} \\
\text { rats }\end{array}$ & SPT, OFT, LDET, TST & $\begin{array}{l}3,9 \mathrm{~g} / \mathrm{kg}, 16 \text { weeks, } \\
\text { i.g.; } 5.9 \mathrm{~g} / \mathrm{kg} \text {, } 2 \text { weeks, } \\
\text { i.g. }\end{array}$ & $\begin{array}{l}\downarrow \text { TNF- } \alpha \text {, IL-1 } \beta, \text { IL-6 } \\
\text { in plasma and } \\
\text { hippocampus }\end{array}$ & $\begin{array}{l}\downarrow \mathrm{CRH}, \mathrm{ACTH} \\
\text { in plasma }\end{array}$ & $\begin{array}{l}{[124,134-} \\
137]\end{array}$ \\
\hline
\end{tabular}


Table 2. Cont.

\begin{tabular}{|c|c|c|c|c|c|c|c|c|}
\hline CHM Formula & $\begin{array}{l}\text { Plant Name/Ratio in Fixed } \\
\text { Combination }\end{array}$ & Daily Human Dose & Animal Model & Behavioral Test & $\begin{array}{l}\text { Administration } \\
\text { Dose/Time/Route of } \\
\text { Treatment }\end{array}$ & $\begin{array}{l}\text { Effects on } \\
\text { Mediators of } \\
\text { Inflammation }\end{array}$ & $\begin{array}{l}\text { Effects on } \\
\text { Hormones of } \\
\text { the HPA Axis }\end{array}$ & Reference \\
\hline Kaixin San & $\begin{array}{l}\text { Panax ginseng C.A. Meyer, Poria } \\
\text { cocos (Schw.) Wolf, Polygala } \\
\text { tenuifolia Willd, and Acorus } \\
\text { tatarinowii Schott.; 1:1:25:50 or } \\
\text { 3:2:2:3 or 1:1:1:2 }\end{array}$ & 2 times/day & $\begin{array}{l}\text { CUMS rats; CUMS } \\
\text { rats }\end{array}$ & SPT & $\begin{array}{l}338,676 \mathrm{mg} / \mathrm{kg}, \\
3 \text { weeks, p.o.; } 3, \\
10 \mathrm{~g} / \mathrm{kg}, 6 \text { weeks, i.g. }\end{array}$ & $\begin{array}{l}\downarrow \text { COX-2, IL-2, IL-6, } \\
\text { TNF- } \alpha \text { in serum } \\
\text { and hippocampus } \\
\uparrow \text { IL-10, IFN- } \gamma \text { in } \\
\text { hippocampus } \\
\text { and serum }\end{array}$ & $\begin{array}{l}\downarrow \mathrm{CRH}, \mathrm{ACTH}, \\
\text { CORT in serum } \\
\text { and organs }\end{array}$ & $\begin{array}{l}{[29,138-} \\
140]\end{array}$ \\
\hline Si-Ni San & $\begin{array}{l}\text { Citrus aurantium L., Bupleurum } \\
\text { chinense DC., Paeonia lactiflora } \\
\text { Pall., and Glycyrrhiza uralensis } \\
\text { Fisch.; 2:2:3:2 }\end{array}$ & 2 times/day & $\begin{array}{l}\text { Reserpine-induced } \\
\text { rats; Mice }\end{array}$ & FST, SPT, OFT, TST & $\begin{array}{l}0.75,1.5,3.0 \mathrm{~g} / \mathrm{kg}, \\
2 \text { weeks, p.o.; } 325,650, \\
1300 \mathrm{mg} / \mathrm{kg}, 60 \mathrm{~min} \\
\text { p.o. }\end{array}$ & $\begin{array}{l}\downarrow \text { IL-1 } \beta, \text { IL-6, TNF- } \alpha \\
\text { in serum, liver, and } \\
\text { hippocampus } \\
\downarrow \text { NF- } \mathrm{B} \text { in } \\
\text { hippocampus }\end{array}$ & $\begin{array}{l}\downarrow \text { CORT } \\
\text { in serum }\end{array}$ & {$[16,17,141]$} \\
\hline $\begin{array}{l}\text { Banxia houpo } \\
\text { Decoction }\end{array}$ & $\begin{array}{l}\text { Pinellia ternate (Thunb.) Breit., } \\
\text { Smilax glabra Roxb, Houpoea } \\
\text { officinalis (Rehder and E. H. } \\
\text { Wilson) N. H. Xia and C. Y. Wu, } \\
\text { Zingiber officinale Roscoe, and } \\
\text { Folium Perillae; } 4: 4: 3: 3: 2\end{array}$ & 2 times/day & CUMS rats & SPT & $\begin{array}{l}3.29,6.58 \mathrm{~g} / \mathrm{kg}, \\
6 \text { weeks, i.g. }\end{array}$ & $\begin{array}{l}\downarrow \text { NLRP3 activation } \\
\text { in livers, } \\
\text { hypothalamus, PFC }\end{array}$ & $\begin{array}{l}\downarrow \text { CORT, CRF } \\
\text { in serum }\end{array}$ & {$[15,142]$} \\
\hline
\end{tabular}

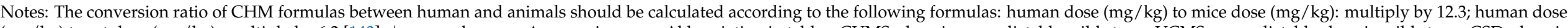

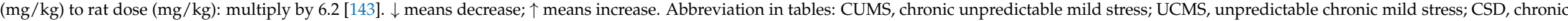

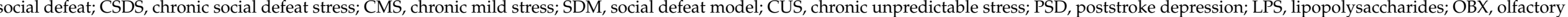

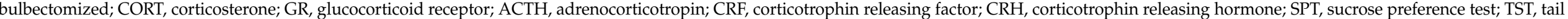

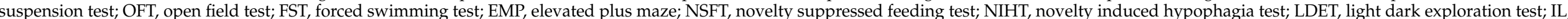

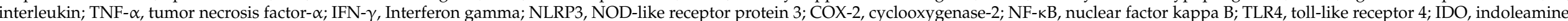

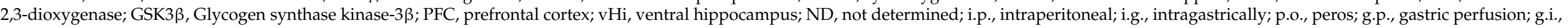
gastric intubation. 


\section{CHM Modulation of the HPA Axis}

It is well known that hyperactivation of the HPA axis in the neuroendocrine system, induced by acute or chronic stress, is a common feature in depressive patients. In response to these somatic stimuli, corticotrophin releasing factor (CRF) or corticotrophin releasing hormone (CRH) is secreted from the median paraventricular nucleus in the hypothalamus and then activates the pituitary to synthesize and release adrenocorticotropic hormone (ACTH). ACTH further activates the adrenal cortex to release glucocorticoid (corticosterone (CORT) or cortisol), which, in turn, regulates the HPA axis through a negative feedback loop at multiple levels: directly on elements of the axis and indirectly through the PFC, amygdala, and hippocampus [144-146] (Figure 1). Studies have demonstrated that hyperactivity of the HPA axis reduces synaptic function, atrophies neurons, and subsequently results in depressive behaviors [147]. Additionally, excessive CRF secretion induced by the desensitization of CRF pituitary receptors, leads to high concentrations of CRF in the CNS, which contributes to the risk of depression $[148,149]$. Moreover, the abnormal activation of the HPA axis can also be induced by the downregulation of glucocorticoid receptors (GR) in the hippocampus [150]. These studies have provided several pharmacological targets to suppress stress-induced hyperactivation of the HPA axis, but attempts to develop novel agents directed toward the HPA axis in the treatment of depression have not been successful.

On the other hand, preclinical studies have demonstrated that many CHM herbal constituents or formulas can attenuate depressive-like symptoms through the modulation of the activity of the HPA axis in depressive animal models [101,102,131-133]. These findings have provided the molecular basis for understanding the mechanism of action of CHM in the treatment of depression, by which CHM constituents or formulas produce antidepressant activities by specifically acting on modulators of the HPA axis. In this section, we review the roles that CHM play in the modulation of the activity of the HPA axis. Several representative $\mathrm{CHM}$ constituents that have been demonstrated to target the hormone receptors in the HPA axis are shown in Figure 3. Other CHM constituents or formulas that modulate the release of HPA axis hormones are listed in Tables 1 and 2, respectively.<smiles></smiles>

Quercetin<smiles>Cc1cc(O)c(=O)c2c(O)c(O)cc(O)c2c1-c1cc2c(O)cc(O)c(O)c2c2c(O)c(O)cc(O)c12</smiles>

Pseudohypericin

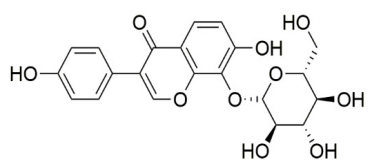

Puerarin

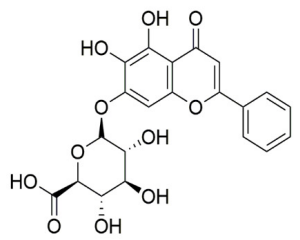

Baicalin<smiles>O=C(/C=C/c1ccc(O)cc1)OCC1OC(O)C(O)C(O)C1Oc1c(-c2ccc(O)cc2)oc2cc(O)cc(O)c2c1=O</smiles>

Tribulus Terrestris Saponins

Figure 3. Several representative $\mathrm{CHM}$ constituents that have been demonstrated to exhibit antidepressant-like activities through their action on HPA axis hormone receptors. 


\subsection{CRF Antagonists}

CRF is commonly considered to be a vital factor in response to stress at the neural, endocrinological, and immunological levels [9]. It is noticeable that CRF is involved in the structural integrity of the brain and in the regulation of neurotransmitter transmission [151] Studies have demonstrated that CRF antagonists could be potential antidepressants that alleviate depressive symptoms through the suppression of the hyperactivation of the HPA axis [152].

Quercetin, a flavonoid abundantly distributed in many herbs, has been shown to exhibit anxiolytic- and antidepressant-like activities in animal models by antagonizing the effect of CRF [153]. In this study, the administration of quercetin (20 or $40 \mathrm{mg} / \mathrm{kg}$, $60 \mathrm{~min}$, p.o.) significantly reduced the levels of CORT and adrenocorticotropic hormone in plasma and the mRNA expression of CRF in the hypothalamic region in water immersion-restraint rats. It also demonstrated that quercetin suppressed CRF expression, probably through the modulation of the DNA-binding activity of the glucocorticoid receptor and the phosphorylation of the cyclic adenosine $3^{\prime}, 5^{\prime}$-monophosphate (cAMP) response element-binding protein and extracellular signal-regulated kinase $1 / 2$ in the hypothalamic region [154]. While this study has provided experimental evidence that quercetin acts as a modulator antagonizing the effect of CRF in the HPA axis, the specific drug-target interaction remains to be uncovered.

\subsection{Corticotrophin Releasing Factor $1\left(C R F_{1}\right)$ Receptor Antagonists}

After its release, $\mathrm{CRF}$ binds to two major receptors: $\mathrm{CRF}_{1}$ and $\mathrm{CRF}_{2}$. It is noteworthy that the $\mathrm{CRF}_{1}$ receptor is widely distributed in the brain, while the $\mathrm{CRF}_{2}$ receptor is highly expressed in peripheral tissues $[9,155]$. Increasing evidence has suggested that an elevated $\mathrm{CRF}_{1}$ receptor function, rather than the $\mathrm{CRF}_{2}$ receptor, is involved in the pathogenesis of anxiety and depression [156]. Furthermore, several lines of preclinical evidence has shown that knockout of the $\mathrm{CRF}_{1}$ receptor in mice produces anti-anxiety effects [9], while $\mathrm{CRF}_{2}$ receptor-deficient mice exhibit increased anxiety- and depressive-like behaviors $[9,157,158]$. Thus, the $\mathrm{CRF}_{1}$ receptor seems to be a key receptor for the HPA axis in the pituitary in response to stress, and the blocking $\mathrm{CRF}_{1}$ receptor has been proposed to be an effective therapeutic approach in depression treatment.

In response to stress, CRF initiates the activity of the HPA axis through by binding to the $\mathrm{CRF}_{1}$ receptor in the anterior pituitary and thus activating adrenocorticotropic hormone secretion [159]. Clinical studies have suggested that the $\mathrm{CRF}_{1}$ receptor plays a crucial role in individuals' risk of developing depression [151]. It is noteworthy that $\mathrm{CRF}_{1}$ receptor antagonists have been tested for their efficacy in depression treatment, but the results were inconsistent. One study has shown that the administration of NBI-30775/R121919 (40-80 mg/day for 30 days), a CRF 1 receptor antagonist, significantly attenuated depressive symptoms in patients [160]. However, in another study, the authors did not observe the antidepressant effects of $\mathrm{CRF}_{1}$ receptor antagonists, such as CP-316311 [161]. It has also been demonstrated that some $\mathrm{CRF}_{1}$ receptor antagonists, including antalarmin, CP154,526, and R121919, did not produce antidepressant-like effects in rat models [162]. These studies have argued that treatment with $\mathrm{CRF}_{1}$ antagonists is only beneficial for depressive patients with CRF overactivity $[160,161]$.

CHM constituents have been shown to produce antidepressant effects in animal or cell models by antagonizing the $\mathrm{CRF}_{1}$ receptor $[9,110,163]$. The activity of three major constituents isolated from St. John's wort, hypericin, pseudohypericin, and hyperforin against the $\mathrm{CRF}_{1}$ receptor has been examined by measuring their effects on CRF-stimulated cAMP formation [163]. This study showed that only pseudohypericin $(10 \mu \mathrm{M})$ selectively inhibits $\mathrm{CRF}_{1}$ receptor activity, but hypericin and hyperforin antagonizes both CRF and calcitonin [163]. To our knowledge, pseudohypericin is the first herbal molecule to be identified as a $\mathrm{CRF}_{1}$ receptor antagonist. 


\subsection{GR Agonists or Antagonists}

GR is a glucocorticoid receptor that is distributed in the HPA axis. Hyperactivation of the HPA axis can impair GR function because of the elevated cortisol and glucocorticoids [164]. Additionally, GR dysfunction may also result from decreased glucocorticoid binding to GR or decreased GR expression in the HPA axis $[165,166]$. It has been observed that, in comparison with healthy controls, GR mRNA levels are decreased in the brain regions of depressed patients in postmortem studies [167,168]. Another study showed that treatment with antidepressants can increase GR binding and GR mRNA expression in the brain, thus ameliorating depressive symptoms [169]. Thus, the upregulation of GR expression and function through, for example, a GR agonist, has been proposed to be pivotal for the therapeutic mechanism of antidepressants. However, a clinical study involving 490 patients with depression indicated that either an increased or decreased GR mRNA results in a greater susceptibility to depression [170]. It is noticeable that polymorphisms of the GR gene play a critical role in the pathogenesis of depression [170]. Hence, GR antagonists have also been recognized to be potential modulators in the development of antidepressants. Clinical studies have shown that a GR antagonist, mifepristone, ameliorates psychotic symptoms and cognitive deficits in patients with depression or bipolar disorders [171,172]. Preclinical studies have also demonstrated that GR deficits in the PFC of mice resulted in depressive-like behaviors [173], which can be ameliorated by the administration of mifepristone [173]. However, in a clinical Phase III study, mifepristone was found to have disappointing effects in terms of the effective reduction of psychotic symptoms in depression sufferers [174], and its abortifacient properties severely compromised its use in women with depression [175]. Thus, mifepristone has not been recognized as an antidepressant drug on the market.

CHM herbal constituents or formulas have been reported to attenuate depressive behaviors through the modulation of stress-impaired GR in animal models. The administration of baicalin $(20 \mathrm{mg} / \mathrm{kg}, 21$ days, p.o.), a major constituent in Scutellaria baicalensis Georgi, has been demonstrated to significantly attenuate CORT-induced behavioral abnormalities through the upregulation of GR mRNA and GR $\alpha$ expression in the hippocampus in mice [122]. In addition, a CHM formula, known as the Huang-Qin-Hua-Shi decoction ( $1 \mathrm{~mL} / 100 \mathrm{~g}, 3$ weeks, i.g.), has also been shown to block the high-temperature- and high-humidity-stress-induced upregulation of hypothalamus GR mRNA expression in rats, which is similar to the action of the GR antagonist, mifepristone [176]. While these studies have demonstrated that $\mathrm{CHM}$ constituents or formulas exert antidepressant-like activities through their actions on GR, the molecular mechanisms and specific acting proteins are still poorly understood.

\section{CHM Effects on the Neuroendocrine-Immune Network}

As mentioned above, either the neuroimmune or neuroendocrine system plays a pivotal role in the pathogenesis of depression, but neither of these individual systems is fully responsible for the pathogenesis of depression. Indeed, clinical studies have demonstrated that abnormal neuroinflammatory responses of the immune system and dysfunction of the HPA axis commonly co-occur in depressive patients [177]. In addition, preclinical evidence has suggested that crosstalk exists between two biological systems through neural, endocrinal, and immunological interactions in the pathogenesis of depression (Figure 1).

Stress activates the HPA axis and sympathetic nervous system, resulting in neuroendocrinal and immunological changes, which, in turn, promote detrimental neuroinflammatory reactions [7,177-181]. Glucocorticoid immunomodulatory action is a key interaction between the HPA axis and neuroimmune system, which allows for coping with any situation that could challenge homoeostasis in the pathogenesis of depression [182,183]. Specifically, glucocorticoids exert immunomodulatory effects, primarily through GR-mediated inflammatory factors, including NF- $\mathrm{KB}$ and activator protein-1 [184-186]. Meanwhile, proinflammatory cytokines can also regulate the HPA axis by disturbing the GR function mediated by inflammatory signaling components, such as p38MAPK, NF- $\mathrm{KB}$, and 
cyclooxygenase-2 (COX2) [8,47]. All MAPKs are potential targets of the anti-inflammatory actions of glucocorticoids through the inhibition of their phosphorylation, whereas proinflammatory cytokines induce the abnormal activation of MAPK signaling, which results in the alternation of GR phosphorylation and activity [8]. Furthermore, a chronic blockade of GR reverses GR dysfunction and decreases depressive-like behaviors induced by LPS $[187,188]$.

Additionally, the activity of the HPA axis is also regulated by proinflammatory cytokines, such as IL-6, IL-1 $\beta$, and TNF- $\alpha$, which can easily cross BBB and exert their effects through various cytokine receptors [9]. In Li's study, elevated CORT levels were observed in the plasma and hippocampus after the administration of LPS [189]. It has also been indicated that an intraperitoneal injection of IL- 1 administered to rats activated the HPA axis by increasing the ACTH and corticosterone levels in plasma [190]. On the other hand, it has been demonstrated that the levels of TNF- $\alpha$ and IL- 6 were upregulated by an intraperitoneal injection of CRF [191]. It is noteworthy that neuroinflammation in stress-induced animal models can be attenuated by the CRF antagonist, SSR125543 [192]. These findings have suggested that the release of CORT, ACTH, and CRF can be induced by proinflammatory cytokines and, conversely, proinflammatory cytokines can also be regulated by the modulation of HPA axis hormones.

The HPA axis has been shown to be involved in microglial activation. Both CRF receptors and GR are abundantly distributed in microglial cells [193-195], and CRF stimulates release of TNF- $\alpha$ in cultured microglial cells [196]. High levels of glucocorticoids have been shown to participate in both proinflammatory cytokine production and the sensitization of microglial cells $[6,197]$. In addition, glucocorticoids induce microglial proliferation in restraint stress-induced mice [197]. However, due to a lack of correlation between the HPA axis and immune measures, the specific function of the HPA axis in microglial physiology and the mechanism by which chronic cytokine exposure influences the HPA axis function remains to be uncovered $[198,199]$. Overall, these studies have indicated that the reciprocal regulation between the HPA axis and neuroimmune system represents a common feature in the pathogenesis of depression.

It has often been reported that a CHM herbal constituent exhibits multiple effects in the pathogenesis of depression [200-202]. Several representative CHM constituents that have been shown to exert multiple actions on the neuroendocrine-immune network are shown in Figure 4. Ginsenoside Rg3 (20 or $40 \mathrm{mg} / \mathrm{kg}$, 3 days, i.g.) was isolated from Panax ginseng C.A. Meyer has been shown to effectively suppress LPS-induced neuroinflammation by reducing the proinflammatory cytokines (IL-1 $\beta$, IL-6, and TNF- $\alpha$ ), NF-KB signal pathway, and microglial activation in the brain [107]. It has also been reported that Rg3 (20 or $40 \mathrm{mg} / \mathrm{kg}, 14$ days, i.g.) attenuated the hyperactivation of the HPA axis by reducing CRH, CORT, and ACTH in CUS rats [108]. Furthermore, it has been shown that total ginsenosides (200 mg/kg, 7 days, i.g.) significantly decrease serum CORT levels, increase GR mRNA expression, and reduce IL-1 $\beta$, IL-6, TNF- $\alpha$, and IDO in the hippocampus of LPS mice or CUMS rats $[104,105]$.

Curcumin, a diarylheptanoid from Curcuma longa L., is another example of CHM constituents that possess multiple actions on the neuroendocrine-immune network. Xu et al. showed that curcumin ( 5 or $10 \mathrm{mg} / \mathrm{kg}$, 21 days, p.o.) produces antidepressant activity by suppressing the aberrant activation of the HPA axis caused by an elevated serum CORT level and GR mRNA expression in CUS rats [116]. Interestingly, a recent study has shown that curcumin $(100 \mathrm{mg} / \mathrm{kg}$, 4 weeks, i.g.) significantly reduces the mRNA expression of proinflammatory cytokines, including IL- $1 \beta$, IL-6, and TNF- $\alpha$, and suppresses the activation of NF- $\mathrm{KB}$ signaling and the NLRP3 inflammasome in CUMS rats [115].

The phenomenon that one CHM constituent exerts multiple actions on several biological systems has been understood poorly because of the lack of experimental evidence to define its pharmacological profiles and specific interactions with its targeting proteins [12]. It is most likely the case that crosstalk exists between these biological systems or that one CHM constituent acts non-selectively on multiple targets [12]. This makes it difficult to 
understand the mechanisms of action of CHM constituents at the molecular level. Thus, more in-depth studies are required to uncover the specific interactions between these CHM constituents and their targeting proteins. Nevertheless, multi-target actions of these CHM constituents provide the scientific basis for interpreting their system-wide mechanisms of action.

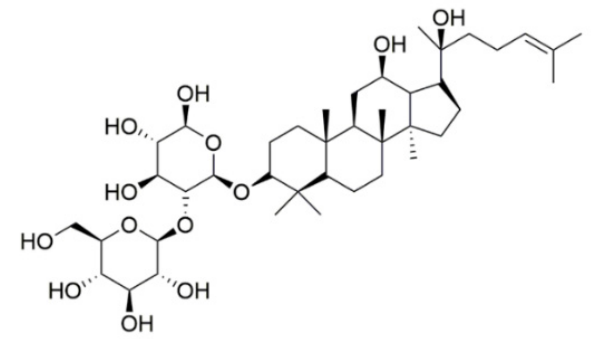

Ginsenoside Rg3<smiles>COc1cc(/C=C/C(=O)CC(=O)/C=C/c2ccc(O)c(OC)c2)ccc1O</smiles>

Curcumin<smiles>OC[C@H]1O[C@@H](OCCc2ccc(O)cc2)[C@H](O)[C@H](O)[C@H]1O</smiles>

Salidroside

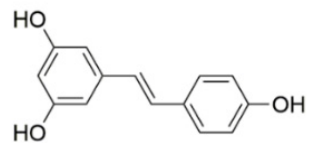

Resveratrol<smiles>O=C(/C=C/c1ccc(O)c2c1C(C(=O)OC(Cc1ccc(O)c(O)c1)C(=O)O)C(c1ccc(O)c(O)c1)O2)OC(Cc1ccc(O)c(O)c1)C(=O)O</smiles>

Salvianolic acid B

Figure 4. Several representative $\mathrm{CHM}$ constituents that have been shown to produce antidepressant effects through their actions on the neuroendocrine-immune network.

In addition to these CHM constituents, many CHM antidepressant formulas have been shown to possess multiple underlying mechanisms of action, particularly on the HPA axis and neuroimmune system (Table 2). This is shown in the studies on Kai-XinSan (KXS), an empirical antidepressant formula, which consists of Panax ginseng C.A. Meyer, Poria cocos (Schw.) Wolf, Polygala tenuifolia Willd, and Acorus tatarinowii Schott [203]. A chronic administration of KXS (338 or $676 \mathrm{mg} / \mathrm{kg}, 3$ weeks, i.g.) has been shown to produce antidepressant-like activity in CUMS-induced animal models through the reduction of COX-2, IL-2, IL-6, and TNF- $\alpha$ expression levels and increase in IFN- $\gamma$ and IL-10 production [29,138,204]. Notably, in other studies, KXS (0.9 or $2.7 \mathrm{~g} / \mathrm{kg}, 5$ weeks, i.g.) has also been reported to modulate the activity of the HPA axis by reversing the elevated ACTH level in CMS-induced mice [138,205]. Taken together, these studies indicate that the underlying mechanisms of KXS, as an antidepressant formula, include its actions on the neuroendocrine-immune network.

In comparison with single CHM herbal constituents, the multidrug feature of a CHM antidepressant formula confers its pharmacological actions on multiple targets toward diverse pathological systems. The antidepressant actions of KXS are triggered by its numerous bioactive constituents within the formula. For instance, ginsenosides Rg1, Rg3, Rh1, Rh3, $\mathrm{Rb1}, \mathrm{Rk1}$, and Rf from Panax ginseng C.A. Meyer have been demonstrated to exhibit dual actions against neuroinflammation and hyperactivation of the HPA axis [106,108,206-215], while 3,6'-disinapoyl sucrose and the oligosaccharide esters-enriched fraction, YZ50, from 
Polygala tenuifolia Willd have been shown to possess bioactivity that de-hyperactivates the HPA axis [216-218]. Additionally, poricoic acid A, isolated from Poria cocos (Schw.) Wolf, has been reported to produce anti-inflammatory effects by inhibiting prostaglandin $\mathrm{E}_{2}$ and NO production through a decrease in COX-2 and iNOS expression, respectively [219]. $\beta$-Asarone, a major bioactive constituent of Acorus tatarinowii Schott, has also been demonstrated to be an anti-inflammation agent, as it downregulates TNF- $\alpha$, IL-1 $\beta$, and IL-6 expression [220]. While the molecular interactions between these herbal constituents and their pharmacological targets remain to be uncovered, multiple actions of these constituents in KXS toward multiple biological systems, such as the neuroendocrine-immune network, represent an excellent example of CHM antidepressant formulas in the systematic treatment of depression. Likewise, many other $\mathrm{CHM}$ antidepressant formulas have been shown to possess multiple mechanisms of action on diverse biological systems, particularly the neuroendocrine-immune network, in the pathogenesis of depression (Table 2).

Dysfunction of the neuroimmune or neuroendocrine system results in profound effects on the CNS through the neuroendocrine-immune network. To uncover the system-wide mechanism of action of KXS, a study has been conducted to assess the protein expression in serum samples of depressive patients, before or after Shen-Zhi-Ling (a proprietary tablet formulated from KXS) treatment (3.2 g/day, 8 weeks, i.g.), using quantitative proteomic analysis [221]. Of a total of 878 serum proteins, the abnormal expression of 12 proteins in depressive patients could be reversed by treatment with KXS. Functional analysis further revealed that these proteins are implicated in platelet activation, immune regulation, and lipid metabolism. Moreover, a quantitative proteomic study has also been performed to evaluate the hippocampal proteins of CMS-induced rats in response to KXS administration (0.6 g/ kg, 14 days, i.g.) [222]. This study identified 33 hippocampal proteins that are associated with KXS treatment. Protein-protein interaction network analysis showed that these proteins can be classified into several categories that participate in glutamate signaling, synaptic plasticity, the metabolic process, the cell survival process, and the BDNF, mTORC1, and cAMP pathways. These studies indicated that KXS exhibits antidepressant actions through targeting numerous proteins across multiple biological systems, providing a network or systems pharmacology approach to understanding the mechanism of action of KXS at the systems level.

\section{Discussion}

Numerous empirical CHM antidepressant formulas are often used in clinical practice for the treatment of depression (Table 2). To elucidate the mechanism of action by which a CHM formula exhibits antidepressant-like activity through the modulation of multiple biological factors across divergent systems is an important research direction. Two major pharmacological approaches are commonly employed in the analysis of the mechanism of action of a CHM formula on the biological factors in the pathogenesis of depression. One is the molecular approach, which uses single bioactive constituents from an herb used in a CHM antidepressant formula to explore their specific actions on potential pharmacological targets. The studies that employ the molecular approach have provided a scientific basis for revealing the mechanism of action of a CHM antidepressant herb or formula at the molecular level (Figures $2-4$ and Table 1). Because the constituent complexity and drugdrug interactions of an entire formula often prevent the molecular mechanism of action from being uncovered, the molecular approach plays a critical role in our understanding of the drug-target interactions in depression treatment. However, the effects of single molecular constituents cannot exactly reflect the action of a CHM composite formula, which contains numerous bioactive constituents that are proposed to simultaneously act on diverse pharmacological targets across biological systems. Hence, it is necessary to integrate the mechanism of action from the molecular level into the systems level in order to understand the role of $\mathrm{CHM}$ in depression treatment.

Another approach is the systems pharmacology approach, which involves uncovering the system-wide mechanism of action of an entire CHM antidepressant formula. Systems 
pharmacology studies drugs, drug targets, and drug effects at the systems level and reveals all responses to the pharmacological actions of drugs across various biological systems [223]. The systems pharmacology approach has recently been applied in studies of CHM antidepressant formulas and shown to be a powerful tool for understanding the system-wide mechanism of action (Table 2 and Section 5). It aims to create a network of the biological factors within a specific system or across diverse systems in response to the pharmacological actions of an entire CHM formula. Several advanced analysis techniques, including DNA or RNA microarray [200,224-228] and quantitative proteomics [229], have been used to identify the potential targeting proteins that are associated with a typical CHM formula. While systems pharmacology-based studies provide a holistic point of view on the pharmacological actions of a given CHM formula, they cannot provide detailed information on molecular drug-target interactions. In addition, the targeting protein candidates resulting from the system pharmacology-based analysis still require further validation by the molecular approaches. While these two approaches are commonly used in preclinical studies, neither can provide a holographic picture of the mechanism of action of a CHM formula in the treatment of depression. Therefore, it is vital to integrate the two approaches into the study of CHM in order to understand the mechanism of action of a CHM antidepressant formula in its entirety.

In summary, the neuroimmune or neuroendocrine system not only exhibit profound effects on the CNS, but also reciprocally regulate one another through the neuroendocrineimmune network. Thus, the effective approach to the treatment of depression induced by the dysfunction of the neuroendocrine-immune network should concurrently target multiple pathological factors across these biological systems. Preclinical studies have demonstrated that the holistic, multidrug, and multitarget CHM represents an excellent example of systems medicine in the treatment of depression. Therefore, we expect that CHM antidepressant formulation will be accepted broadly as an effective medication for the systematic treatment of depression.

Author Contributions: C.L. wrote the manuscript and prepared the figures and tables. B.H. assisted in the collection of references and preparation of figures. Y.-W.Z. revised and edited the manuscript. All authors have read and agreed to the published version of the manuscript.

Funding: This research was funded by the China Postdoctoral Science Foundation [2019M662844]; this research was also funded by the Guangdong Basic and Applied Basic Research Foundation [2019A1515011569] and National Natural Science Foundation of China [32071233].

Institutional Review Board Statement: Not applicable.

Informed Consent Statement: Not applicable.

Data Availability Statement: No new data were created or analyzed in this study. Data sharing is not applicable to this article.

Acknowledgments: C.L. was financially supported by the International Training Program for Outstanding Scientific Postdoctoral Research of Guangdong Province, China.

Conflicts of Interest: The authors declare no conflict of interest.

\section{References}

1. WHO. Depression. Available online: https://www.who.int/news-room/fact-sheets/detail/depression (accessed on 30 January 2020).

2. Pothula, S.; Kato, T.; Liu, R.J.; Wu, M.; Gerhard, D.; Shinohara, R.; Sliby, A.N.; Chowdhury, G.M.; Behar, K.L.; Sanacora, G.; et al. Cell-Type specific modulation of NMDA receptors triggers antidepressant actions. Mol. Psychiatry 2020. [CrossRef] [PubMed]

3. Jiang, C.Y.; Qin, X.Y.; Yuan, M.M.; Lu, G.J.; Cheng, Y. 2,3,5,4'-Tetrahydroxystilbene-2-O- $\beta$-D-glucoside Reverses Stress-Induced Depression via Inflammatory and Oxidative Stress Pathways. Oxid. Med. Cell. Longev. 2018, 2018, 9501427. [CrossRef] [PubMed]

4. Zhang, Y.W.; Cheng, Y.C. Challenge and Prospect of Traditional Chinese Medicine in Depression Treatment. Front. Neurosci. 2019, 13, 190. [CrossRef] [PubMed]

5. Fogaca, M.V.; Duman, R.S. Cortical GABAergic Dysfunction in Stress and Depression: New Insights for Therapeutic Interventions. Front. Cell. Neurosci. 2019, 13, 87. [CrossRef] 
6. Kim, Y.K.; Na, K.S.; Myint, A.M.; Leonard, B.E. The role of pro-inflammatory cytokines in neuroinflammation, neurogenesis and the neuroendocrine system in major depression. Prog. Neuropsychopharmacol. Biol. Psychiatry 2016, 64, 277-284. [CrossRef]

7. Ignacio, Z.M.; da Silva, R.S.; Plissari, M.E.; Quevedo, J.; Reus, G.Z. Physical Exercise and Neuroinflammation in Major Depressive Disorder. Mol. Neurobiol. 2019, 56, 8323-8335. [CrossRef]

8. Zunszain, P.A.; Anacker, C.; Cattaneo, A.; Carvalho, L.A.; Pariante, C.M. Glucocorticoids, cytokines and brain abnormalities in depression. Prog. Neuropsychopharmacol. Biol. Psychiatry 2011, 35, 722-729. [CrossRef]

9. Jiang, Y.Z.; Peng, T.M.; Gaur, U.; Silva, M.; Little, P.; Chen, Z.; Qiu, W.; Zhang, Y.D.; Zheng, W.H. Role of Corticotropin Releasing Factor in the Neuroimmune Mechanisms of Depression: Examination of Current Pharmaceutical and Herbal Therapies. Front. Cell. Neurosci. 2019, 13, 290. [CrossRef]

10. Wang, Y.; Li, M.; Liang, Y.; Yang, Y.; Liu, Z.; Yao, K.; Chen, Z.; Zhai, S. Chinese Herbal Medicine for the Treatment of Depression: Applications, Efficacies and Mechanisms. Curr. Pharm. Des. 2017, 23, 5180-5190. [CrossRef]

11. Feng, D.D.; Tang, T.; Lin, X.P.; Yang, Z.Y.; Yang, S.; Xia, Z.A.; Wang, Y.; Zheng, P.; Wang, Y.; Zhang, C.H. Nine traditional Chinese herbal formulas for the treatment of depression: An ethnopharmacology, phytochemistry, and pharmacology review. Neuropsychiatr. Dis. Treat. 2016, 12, 2387-2402. [CrossRef]

12. Li, C.; Huang, J.; Cheng, Y.C.; Zhang, Y.W. Traditional Chinese Medicine in Depression Treatment: From Molecules to Systems. Front. Pharmacol. 2020, 11, 586. [CrossRef] [PubMed]

13. Chen, G.; Guo, X. Neurobiology of Chinese Herbal Medicine on Major Depressive Disorder. Int. Rev. Neurobiol. 2017, 135, 77-95. [CrossRef] [PubMed]

14. Yeung, W.F.; Chung, K.F.; Ng, K.Y.; Yu, Y.M.; Zhang, S.P.; Ng, B.F.; Ziea, E.T. Prescription of Chinese Herbal Medicine in Pattern-Based Traditional Chinese Medicine Treatment for Depression: A Systematic Review. Evid. Based Complement. Altern. Med. 2015, 2015, 160189. [CrossRef] [PubMed]

15. Jia, K.K.; Zheng, Y.J.; Zhang, Y.X.; Liu, J.H.; Jiao, R.Q.; Pan, Y.; Kong, L.D. Banxia-Houpu decoction restores glucose intolerance in CUMS rats through improvement of insulin signaling and suppression of NLRP3 inflammasome activation in liver and brain. J. Ethnopharmacol. 2017, 209, 219-229. [CrossRef] [PubMed]

16. Yi, L.T.; Li, J.; Liu, B.B.; Li, C.F. Screening of the antidepressant-like effect of the traditional Chinese medicinal formula Si-Ni-San and their possible mechanism of action in mice. Pharmacogn. Res. 2013, 5, 36-42. [CrossRef] [PubMed]

17. Zong, Y.; Chen, T.; Dong, H.; Zhu, L.; Ju, W. Si-Ni-San Prevents Reserpine-Induced Depression by Inhibiting Inflammation and Regulating CYP450 Enzymatic Activity. Front. Pharmacol. 2019, 10, 1518. [CrossRef]

18. Smith, R.S. The macrophage theory of depression. Med. Hypotheses 1991, 35, 298-306. [CrossRef]

19. Wohleb, E.S.; Franklin, T.; Iwata, M.; Duman, R.S. Integrating neuroimmune systems in the neurobiology of depression. Nat. Rev. Neurosci. 2016, 17, 497-511. [CrossRef]

20. Kohler, O.; Krogh, J.; Mors, O.; Benros, M.E. Inflammation in Depression and the Potential for Anti-Inflammatory Treatment. Curr. Neuropharmacol. 2016, 14, 732-742. [CrossRef]

21. Zhang, J.C.; Yao, W.; Hashimoto, K. Brain-Derived Neurotrophic Factor (BDNF)-TrkB Signaling in Inflammation-Related Depression and Potential Therapeutic Targets. Curr. Neuropharmacol. 2016, 14, 721-731. [CrossRef]

22. Halaris, A. Inflammation and depression but where does the inflammation come from? Curr. Opin. Psychiatry 2019, 32, 422-428. [CrossRef] [PubMed]

23. Tian, H.; Li, G.; Xu, G.; Liu, J.; Wan, X.; Zhang, J.; Xie, S.; Cheng, J.; Gao, S. Inflammatory cytokines derived from peripheral blood contribute to the modified electroconvulsive therapy-induced cognitive deficits in major depressive disorder. Eur. Arch. Psychiatry Clin. Neurosci. 2020. [CrossRef] [PubMed]

24. Ng, A.; Tam, W.W.; Zhang, M.W.; Ho, C.S.; Husain, S.F.; McIntyre, R.S.; Ho, R.C. IL-1 $\beta$, IL-6, TNF- $\alpha$ and CRP in Elderly Patients with Depression or Alzheimer's disease: Systematic Review and Meta-Analysis. Sci. Rep. 2018, 8, 12050. [CrossRef] [PubMed]

25. D'Mello, C.; Swain, M.G. Immune-to-Brain Communication Pathways in Inflammation-Associated Sickness and Depression. Curr. Top. Behav. Neurosci. 2017, 31, 73-94. [CrossRef] [PubMed]

26. Chiu, W.C.; Su, Y.P.; Su, K.P.; Chen, P.C. Recurrence of depressive disorders after interferon-induced depression. Transl. Psychiatry 2017, 7, e1026. [CrossRef]

27. Zhao, Y.; Shang, P.; Wang, M.; Xie, M.; Liu, J. Neuroprotective Effects of Fluoxetine Against Chronic Stress-Induced Neural Inflammation and Apoptosis: Involvement of the p38 Activity. Front. Physiol. 2020, 11, 351. [CrossRef]

28. Wang, M.; Yan, S.; Zhou, Y.; Xie, P. trans-Cinnamaldehyde Reverses Depressive-Like Behaviors in Chronic Unpredictable Mild Stress Rats by Inhibiting NF-кB/NLRP3 Inflammasome Pathway. Evid. Based Complement. Altern. Med. 2020, $2020,4572185$. [CrossRef]

29. Dong, X.Z.; Wang, D.X.; Lu, Y.P.; Yuan, S.; Liu, P.; Hu, Y. Antidepressant effects of Kai-Xin-San in fluoxetine-resistant depression rats. Braz. J. Med. Biol. Res. 2017, 50, e6161. [CrossRef]

30. Dantzer, R.; O'Connor, J.C.; Lawson, M.A.; Kelley, K.W. Inflammation-Associated depression: From serotonin to kynurenine. Psychoneuroendocrinology 2011, 36, 426-436. [CrossRef]

31. Zhang, Z.; Song, Z.; Shen, F.; Xie, P.; Wang, J.; Zhu, A.S.; Zhu, G. Ginsenoside Rg1 Prevents PTSD-Like Behaviors in Mice Through Promoting Synaptic Proteins, Reducing Kir4.1 and TNF- $\alpha$ in the Hippocampus. Mol. Neurobiol. 2020, 1-14. [CrossRef]

32. Miller, A.H.; Raison, C.L. The role of inflammation in depression: From evolutionary imperative to modern treatment target. Nat. Rev. Immunol. 2016, 16, 22-34. [CrossRef] [PubMed] 
33. Turkheimer, F.E.; Althubaity, N.; Schubert, J.; Nettis, M.A.; Cousins, O.; Dima, D.; Mondelli, V.; Bullmore, E.T.; Pariante, C.; Veronese, M. Increased serum peripheral C-reactive protein is associated with reduced brain barriers permeability of TSPO radioligands in healthy volunteers and depressed patients: Implications for inflammation and depression. Brain Behav. Immun. 2020, 91, 487-497. [CrossRef] [PubMed]

34. Rosenblat, J.D.; Cha, D.S.; Mansur, R.B.; McIntyre, R.S. Inflamed moods: A review of the interactions between inflammation and mood disorders. Prog. Neuropsychopharmacol. Biol. Psychiatry 2014, 53, 23-34. [CrossRef] [PubMed]

35. Duman, R.S.; Aghajanian, G.K.; Sanacora, G.; Krystal, J.H. Synaptic plasticity and depression: New insights from stress and rapid-acting antidepressants. Nat. Med. 2016, 22, 238-249. [CrossRef] [PubMed]

36. Deng, S.L.; Chen, J.G.; Wang, F. Microglia: A Central Player in Depression. Curr. Med. Sci. 2020, 40, 391-400. [CrossRef]

37. Torres-Platas, S.G.; Cruceanu, C.; Chen, G.G.; Turecki, G.; Mechawar, N. Evidence for increased microglial priming and macrophage recruitment in the dorsal anterior cingulate white matter of depressed suicides. Brain Behav. Immun. 2014, 42, 50-59. [CrossRef]

38. Setiawan, E.; Wilson, A.A.; Mizrahi, R.; Rusjan, P.M.; Miler, L.; Rajkowska, G.; Suridjan, I.; Kennedy, J.L.; Rekkas, P.V.; Houle, S.; et al. Role of translocator protein density, a marker of neuroinflammation, in the brain during major depressive episodes. JAMA Psychiatry 2015, 72, 268-275. [CrossRef]

39. Zhang, L.; Zhang, J.; You, Z. Switching of the Microglial Activation Phenotype Is a Possible Treatment for Depression Disorder. Front. Cell. Neurosci. 2018, 12, 306. [CrossRef]

40. Brown, G.C. The endotoxin hypothesis of neurodegeneration. J. Neuroinflamm. 2019, 16, 180. [CrossRef]

41. Bollinger, J.L.; Wohleb, E.S. The formative role of microglia in stress-induced synaptic deficits and associated behavioral consequences. Neurosci. Lett. 2019, 711, 134369. [CrossRef]

42. Wohleb, E.S.; Terwilliger, R.; Duman, C.H.; Duman, R.S. Stress-Induced Neuronal Colony Stimulating Factor 1 Provokes Microglia-Mediated Neuronal Remodeling and Depressive-Like Behavior. Biol. Psychiatry 2018, 83, 38-49. [CrossRef] [PubMed]

43. Wang, Y.S.; Shen, C.Y.; Jiang, J.G. Antidepressant active ingredients from herbs and nutraceuticals used in TCM: Pharmacological mechanisms and prospects for drug discovery. Pharmacol. Res. 2019, 150, 104520. [CrossRef]

44. Guan, F.; Lam, W.; Hu, R.; Kim, Y.K.; Han, H.; Cheng, Y.C. Majority of Chinese Medicine Herb Category “Qing Re Yao" Have Multiple Mechanisms of Anti-inflammatory Activity. Sci. Rep. 2018, 8, 7416. [CrossRef]

45. Lu, Z.B.; Ou, J.Y.; Cao, H.H.; Liu, J.S.; Yu, L.Z. Heat-Clearing Chinese Medicines in Lipopolysaccharide-Induced Inflammation. Chin. J. Integr. Med. 2020, 26, 552-559. [CrossRef]

46. Panossian, A.; Brendler, T. The Role of Adaptogens in Prophylaxis and Treatment of Viral Respiratory Infections. Pharmaceuticals 2020, 13, 236. [CrossRef] [PubMed]

47. Adzic, M.; Brkic, Z.; Mitic, M.; Francija, E.; Jovicic, M.J.; Radulovic, J.; Maric, N.P. Therapeutic Strategies for Treatment of Inflammation-related Depression. Curr. Neuropharmacol. 2018, 16, 176-209. [CrossRef] [PubMed]

48. Young, J.J.; Bruno, D.; Pomara, N. A review of the relationship between proinflammatory cytokines and major depressive disorder. J. Affect. Disord. 2014, 169, 15-20. [CrossRef] [PubMed]

49. Zhang, Z.D.; Li, H.; Wan, F.; Su, X.Y.; Lu, Y.; Chen, D.F.; Zhang, Y.Y. Polysaccharides extracted from the roots of Bupleurum chinense DC modulates macrophage functions. Chin. J. Nat. Med. 2017, 15, 889-898. [CrossRef]

50. Shiu, L.Y.; Huang, H.H.; Chen, C.Y.; Cheng, H.Y.; Chen, C.I.; Kuo, S.M. Reparative and toxicity-reducing effects of liposomeencapsulated saikosaponin in mice with liver fibrosis. Biosci. Rep. 2020, 40, BSR20201219. [CrossRef]

51. Zhang, B.Z.; Guo, X.T.; Chen, J.W.; Zhao, Y.; Cong, X.; Jiang, Z.L.; Cao, R.F.; Cui, K.; Gao, S.S.; Tian, W.R. Saikosaponin-D attenuates heat stress-induced oxidative damage in LLC-PK1 cells by increasing the expression of anti-oxidant enzymes and HSP72. Am. J. Chin. Med. 2014, 42, 1261-1277. [CrossRef]

52. Su, J.; Pan, Y.W.; Wang, S.Q.; Li, X.Z.; Huang, F.; Ma, S.P. Saikosaponin-d attenuated lipopolysaccharide-induced depressive-like behaviors via inhibiting microglia activation and neuroinflammation. Int. Immunopharmacol. 2020, 80, 106181. [CrossRef] [PubMed]

53. Chen, X.Q.; Chen, S.J.; Liang, W.N.; Wang, M.; Li, C.F.; Wang, S.S.; Dong, S.Q.; Yi, L.T.; Li, C.D. Saikosaponin A attenuates perimenopausal depression-like symptoms by chronic unpredictable mild stress. Neurosci. Lett. 2018, 662, 283-289. [CrossRef]

54. Li, J.; Zhao, R.; Li, X.; Sun, W.; Qu, M.; Tang, Q.; Yang, X.; Zhang, S. Shen-Qi-Jie-Yu-Fang exerts effects on a rat model of postpartum depression by regulating inflammatory cytokines and $\mathrm{CD} 4{ }^{+} \mathrm{CD} 25^{+}$regulatory T cells. Neuropsychiatr. Dis. Treat. 2016, 12, 883-896. [CrossRef] [PubMed]

55. Su, W.J.; Zhang, Y.; Chen, Y.; Gong, H.; Lian, Y.J.; Peng, W.; Liu, Y.Z.; Wang, Y.X.; You, Z.L.; Feng, S.J.; et al. NLRP3 gene knockout blocks NF-kB and MAPK signaling pathway in CUMS-induced depression mouse model. Behav. Brain Res. 2017, 322, 1-8. [CrossRef] [PubMed]

56. Ji, H.F.; Zhuang, Q.S.; Shen, L. Genetic overlap between type 2 diabetes and major depressive disorder identified by bioinformatics analysis. Oncotarget 2016, 7, 17410-17414. [CrossRef]

57. Wang, M.; Zhou, W.; Zhou, X.; Zhuang, F.; Chen, Q.; Li, M.; Ma, T.; Gu, S. Antidepressant-Like effects of alarin produced by activation of TrkB receptor signaling pathways in chronic stress mice. Behav. Brain Res. 2015, 280, 128-140. [CrossRef]

58. Masson, J.; Emerit, M.B.; Hamon, M.; Darmon, M. Serotonergic signaling: Multiple effectors and pleiotropic effects. Wiley Interdiscip. Rev. Membr. Transp. Signal. 2012, 1, 685-713. [CrossRef] 
59. Duric, V.; Banasr, M.; Licznerski, P.; Schmidt, H.D.; Stockmeier, C.A.; Simen, A.A.; Newton, S.S.; Duman, R.S. A negative regulator of MAP kinase causes depressive behavior. Nat. Med. 2010, 16, 1328-1332. [CrossRef]

60. Welcome, M.O.; Mastorakis, N.E. Stress-Induced blood brain barrier disruption: Molecular mechanisms and signaling pathways. Pharmacol. Res. 2020, 157, 104769. [CrossRef] [PubMed]

61. Chiang, J.J.; Cole, S.W.; Bower, J.E.; Irwin, M.R.; Taylor, S.E.; Arevalo, J.; Fuligni, A. Depressive symptoms and immune transcriptional profiles in late adolescents. Brain Behav. Immun. 2019, 80, 163-169. [CrossRef]

62. Liu, W.; Jiang, H.L.; Cai, L.L.; Yan, M.; Dong, S.J.; Mao, B. Tanreqing Injection Attenuates Lipopolysaccharide-Induced Airway Inflammation through MAPK/NF-kB Signaling Pathways in Rats Model. Evid. Based Complement. Altern. Med. 2016, 2016, 5292346. [CrossRef] [PubMed]

63. Shih, R.H.; Wang, C.Y.; Yang, C.M. NF-кB Signaling Pathways in Neurological Inflammation: A Mini Review. Front. Mol. Neurosci. 2015, 8, 77. [CrossRef] [PubMed]

64. Lopez-Pedrajas, R.; Ramirez-Lamelas, D.T.; Muriach, B.; Sanchez-Villarejo, M.V.; Almansa, I.; Vidal-Gil, L.; Romero, F.J.; Barcia, J.M.; Muriach, M. Cocaine promotes oxidative stress and microglial-macrophage activation in rat cerebellum. Front. Cell. Neurosci. 2015, 9, 279. [CrossRef] [PubMed]

65. Kaltschmidt, B.; Kaltschmidt, C. NF-кB in the nervous system. Cold Spring Harb. Perspect. Biol. 2009, 1, a001271. [CrossRef] [PubMed]

66. Caviedes, A.; Lafourcade, C.; Soto, C.; Wyneken, U. BDNF/NF-кB Signaling in the Neurobiology of Depression. Curr. Pharm. Des. 2017, 23, 3154-3163. [CrossRef]

67. Pradere, J.P.; Hernandez, C.; Koppe, C.; Friedman, R.A.; Luedde, T.; Schwabe, R.F. Negative regulation of NF-kB p65 activity by serine 536 phosphorylation. Sci. Signal. 2016, 9, ra85. [CrossRef]

68. Koo, J.W.; Russo, S.J.; Ferguson, D.; Nestler, E.J.; Duman, R.S. Nuclear factor- $\mathrm{kB}$ is a critical mediator of stress-impaired neurogenesis and depressive behavior. Proc. Natl. Acad. Sci. USA 2010, 107, 2669-2674. [CrossRef]

69. Bottcher, M.; Muller-Fielitz, H.; Sundaram, S.M.; Gallet, S.; Neve, V.; Shionoya, K.; Zager, A.; Quan, N.; Liu, X.; Schmidt-Ullrich, R.; et al. NF-KB signaling in tanycytes mediates inflammation-induced anorexia. Mol. Metab. 2020, 39, 101022. [CrossRef]

70. Jin, Y.; Cui, R.; Zhao, L.; Fan, J.; Li, B. Mechanisms of Panax ginseng action as an antidepressant. Cell Prolif. 2019, 52, e12696. [CrossRef]

71. Lou, T.; Huang, Q.; Su, H.; Zhao, D.; Li, X. Targeting Sirtuin 1 signaling pathway by ginsenosides. J. Ethnopharmacol. 2020, 268, 113657. [CrossRef]

72. Jiang, N.; Lv, J.; Wang, H.; Huang, H.; Wang, Q.; Lu, C.; Zeng, G.; Liu, X.M. Ginsenoside Rg1 ameliorates chronic social defeat stress-induced depressive-like behaviors and hippocampal neuroinflammation. Life Sci. 2020, 252, 117669. [CrossRef] [PubMed]

73. Fan, C.; Song, Q.; Wang, P.; Li, Y.; Yang, M.; Yu, S.Y. Neuroprotective Effects of Ginsenoside-Rg1 Against Depression-Like Behaviors via Suppressing Glial Activation, Synaptic Deficits, and Neuronal Apoptosis in Rats. Front. Immunol. 2018, 9, 2889. [CrossRef] [PubMed]

74. Hu, J.F.; Song, X.Y.; Chu, S.F.; Chen, J.; Ji, H.J.; Chen, X.Y.; Yuan, Y.H.; Han, N.; Zhang, J.T.; Chen, N.H. Inhibitory effect of ginsenoside Rg1 on lipopolysaccharide-induced microglial activation in mice. Brain Res. 2011, 1374, 8-14. [CrossRef] [PubMed]

75. Kaufmann, F.N.; Costa, A.P.; Ghisleni, G.; Diaz, A.P.; Rodrigues, A.L.S.; Peluffo, H.; Kaster, M.P. NLRP3 inflammasome-driven pathways in depression: Clinical and preclinical findings. Brain Behav. Immun. 2017, 64, 367-383. [CrossRef] [PubMed]

76. Arbore, G.; Kemper, C. A novel "complement-metabolism-inflammasome axis" as a key regulator of immune cell effector function. Eur. J. Immunol. 2016, 46, 1563-1573. [CrossRef]

77. Walsh, J.G.; Muruve, D.A.; Power, C. Inflammasomes in the CNS. Nat. Rev. Neurosci. 2014, 15, 84-97. [CrossRef]

78. Shao, B.Z.; Cao, Q.; Liu, C. Targeting NLRP3 Inflammasome in the Treatment of CNS Diseases. Front. Mol. Neurosci. 2018, 11, 320. [CrossRef]

79. Alcocer-Gómez, E.; de Miguel, M.; Casas-Barquero, N.; Núñez-Vasco, J.; Sánchez-Alcazar, J.A.; Fernández-Rodríguez, A.; Cordero, M.D. NLRP3 inflammasome is activated in mononuclear blood cells from patients with major depressive disorder. Brain Behav. Immun. 2014, 36, 111-117. [CrossRef]

80. Zhang, Y.; Liu, L.; Liu, Y.Z.; Shen, X.L.; Wu, T.Y.; Zhang, T.; Wang, W.; Wang, Y.X.; Jiang, C.L. NLRP3 Inflammasome Mediates Chronic Mild Stress-Induced Depression in Mice via Neuroinflammation. Int. J. Neuropsychopharmacol. 2015, 18, pyv006. [CrossRef]

81. Zhang, Y.; Liu, L.; Peng, Y.L.; Liu, Y.Z.; Wu, T.Y.; Shen, X.L.; Zhou, J.R.; Sun, D.Y.; Huang, A.J.; Wang, X.; et al. Involvement of inflammasome activation in lipopolysaccharide-induced mice depressive-like behaviors. CNS Neurosci. Ther. 2014, 20, 119-124. [CrossRef]

82. Fu, Y.; Yang, P.; Zhao, Y.; Zhang, L.; Zhang, Z.; Dong, X.; Wu, Z.; Xu, Y.; Chen, Y. trans-Cinnamaldehyde Inhibits Microglial Activation and Improves Neuronal Survival against Neuroinflammation in BV2 Microglial Cells with Lipopolysaccharide Stimulation. Evid. Based Complement. Altern. Med. 2017, 2017, 4730878. [CrossRef] [PubMed]

83. Liu, B.; Xu, C.; Wu, X.; Liu, F.; Du, Y.; Sun, J.; Tao, J.; Dong, J. Icariin exerts an antidepressant effect in an unpredictable chronic mild stress model of depression in rats and is associated with the regulation of hippocampal neuroinflammation. Neuroscience 2015, 294, 193-205. [CrossRef] [PubMed] 
84. Chen, Z.; Huang, C.; He, H.; Ding, W. 2, 3, 5, 4'-Tetrahydroxystilbene-2-O- $\beta$-D-glucoside prevention of lipopolysaccharideinduced depressive-like behaviors in mice involves neuroinflammation and oxido-nitrosative stress inhibition. Behav. Pharmacol. 2017, 28, 365-374. [CrossRef] [PubMed]

85. Bian, X.; Liu, X.; Liu, J.; Zhao, Y.; Li, H.; Cai, E.; Li, P.; Gao, Y. Study on antidepressant activity of chiisanoside in mice. Int. Immunopharmacol. 2018, 57, 33-42. [CrossRef]

86. Li, H.; Lin, S.; Qin, T.; Li, H.; Ma, Z.; Ma, S. Senegenin exerts anti-depression effect in mice induced by chronic un-predictable mild stress via inhibition of NF-kB regulating NLRP3 signal pathway. Int. Immunopharmacol. 2017, 53, 24-32. [CrossRef]

87. Wang, H.; Zhang, R.; Qiao, Y.; Xue, F.; Nie, H.; Zhang, Z.; Wang, Y.; Peng, Z.; Tan, Q. Gastrodin ameliorates depression-like behaviors and up-regulates proliferation of hippocampal-derived neural stem cells in rats: Involvement of its anti-inflammatory action. Behav. Brain Res. 2014, 266, 153-160. [CrossRef]

88. Liu, P.; Bai, X.; Zhang, T.; Zhou, L.; Li, J.; Zhang, L. The protective effect of Lonicera japonica polysaccharide on mice with depression by inhibiting NLRP3 inflammasome. Ann. Transl. Med. 2019, 7, 811. [CrossRef]

89. Geng, J.; Liu, J.; Yuan, X.; Liu, W.; Guo, W. Andrographolide triggers autophagy-mediated inflammation inhibition and attenuates chronic unpredictable mild stress (CUMS)-induced depressive-like behavior in mice. Toxicol. Appl. Pharmacol. 2019, $379,114688$. [CrossRef]

90. Zhang, B.; Wang, P.P.; Hu, K.L.; Li, L.N.; Yu, X.; Lu, Y.; Chang, H.S. Antidepressant-Like Effect and Mechanism of Action of Honokiol on the Mouse Lipopolysaccharide (LPS) Depression Model. Molecules 2019, 24, 2035. [CrossRef]

91. Zhang, L.; Previn, R.; Lu, L.; Liao, R.F.; Jin, Y.; Wang, R.K. Crocin, a natural product attenuates lipopolysaccharide-induced anxiety and depressive-like behaviors through suppressing NF-kB and NLRP3 signaling pathway. Brain Res. Bull. 2018, 142, 352-359. [CrossRef]

92. Song, Y.; Sun, R.; Ji, Z.; Li, X.; Fu, Q.; Ma, S. Perilla aldehyde attenuates CUMS-induced depressive-like behaviors via regulating TXNIP/TRX/NLRP3 pathway in rats. Life Sci. 2018, 206, 117-124. [CrossRef] [PubMed]

93. Ji, W.W.; Wang, S.Y.; Ma, Z.Q.; Li, R.P.; Li, S.S.; Xue, J.S.; Li, W.; Niu, X.X.; Yan, L.; Zhang, X.; et al. Effects of perillaldehyde on alternations in serum cytokines and depressive-like behavior in mice after lipopolysaccharide administration. Pharmacol. Biochem. Behav. 2014, 116, 1-8. [CrossRef] [PubMed]

94. Ji, W.W.; Li, R.P.; Li, M.; Wang, S.Y.; Zhang, X.; Niu, X.X.; Li, W.; Yan, L.; Wang, Y.; Fu, Q.; et al. Antidepressant-Like effect of essential oil of Perilla frutescens in a chronic, unpredictable, mild stress-induced depression model mice. Chin. J. Nat. Med. 2014, 12, 753-759. [CrossRef]

95. Park, S.H.; Jang, S.; Son, E.; Lee, S.W.; Park, S.D.; Sung, Y.Y.; Kim, H.K. Polygonum aviculare L. extract reduces fatigue by inhibiting neuroinflammation in restraint-stressed mice. Phytomedicine 2018, 42, 180-189. [CrossRef] [PubMed]

96. Li, C.F.; Chen, X.Q.; Chen, S.M.; Chen, X.M.; Geng, D.; Liu, Q.; Yi, L.T. Evaluation of the toxicological properties and antiinflammatory mechanism of Hemerocallis citrina in LPS-induced depressive-like mice. Biomed. Pharmacother. 2017, 91, 167-173. [CrossRef] [PubMed]

97. Liu, Y.M.; Shen, J.D.; Xu, L.P.; Li, H.B.; Li, Y.C.; Yi, L.T. Ferulic acid inhibits neuro-inflammation in mice exposed to chronic unpredictable mild stress. Int. Immunopharmacol. 2017, 45, 128-134. [CrossRef]

98. Li, J.; Huang, S.; Huang, W.; Wang, W.; Wen, G.; Gao, L.; Fu, X.; Wang, M.; Liang, W.; Kwan, H.Y.; et al. Paeoniflorin ameliorates interferon- $\alpha$-induced neuroinflammation and depressive-like behaviors in mice. Oncotarget 2017, 8, 8264-8282. [CrossRef]

99. An, L.; Li, J.; Yu, S.T.; Xue, R.; Yu, N.J.; Chen, H.X.; Zhang, L.M.; Zhao, N.; Li, Y.F.; Zhang, Y.Z. Effects of the total flavonoid extract of Xiaobuxin-Tang on depression-like behavior induced by lipopolysaccharide and proinflammatory cytokine levels in mice. $J$. Ethnopharmacol. 2015, 163, 83-87. [CrossRef]

100. Zhao, Y.; Zhang, Y.; Pan, F. The effects of EGb761 on lipopolysaccharide-induced depressive-like behaviour in C57BL/6J mice. Cent. Eur. J. Immunol. 2015, 40, 11-17. [CrossRef]

101. Qiu, Z.K.; Zhang, G.H.; Zhong, D.S.; He, J.L.; Liu, X.; Chen, J.S.; Wei, D.N. Puerarin ameliorated the behavioral deficits induced by chronic stress in rats. Sci. Rep. 2017, 7, 6266. [CrossRef]

102. Wang, Z.; Zhang, D.; Hui, S.; Zhang, Y.; Hu, S. Effect of tribulus terrestris saponins on behavior and neuroendocrine in chronic mild stress depression rats. J. Tradit. Chin. Med. 2013, 33, 228-232. [CrossRef]

103. Wang, J.M.; Pei, L.X.; Zhang, Y.Y.; Cheng, Y.X.; Niu, C.L.; Cui, Y.; Feng, W.S.; Wang, G.F. Ethanol extract of Rehmannia glutinosa exerts antidepressant-like effects on a rat chronic unpredictable mild stress model by involving monoamines and BDNF. Metab. Brain Dis. 2018, 33, 885-892. [CrossRef]

104. Kang, A.; Hao, H.; Zheng, X.; Liang, Y.; Xie, Y.; Xie, T.; Dai, C.; Zhao, Q.; Wu, X.; Xie, L.; et al. Peripheral anti-inflammatory effects explain the ginsenosides paradox between poor brain distribution and anti-depression efficacy. J. Neuroinflamm. 2011, 8, 100. [CrossRef] [PubMed]

105. Liu, L.; Luo, Y.; Zhang, R.; Guo, J. Effects of ginsenosides on hypothalamic-pituitary-adrenal function and brain-derived neurotrophic factor in rats exposed to chronic unpredictable mild stress. Zhongguo Zhongyao Zazhi 2011, 36, 1342-1347. [PubMed]

106. Mou, Z.; Huang, Q.; Chu, S.F.; Zhang, M.J.; Hu, J.F.; Chen, N.H.; Zhang, J.T. Antidepressive effects of ginsenoside Rg1 via regulation of HPA and HPG axis. Biomed. Pharmacother. 2017, 92, 962-971. [CrossRef]

107. Kang, A.; Xie, T.; Zhu, D.; Shan, J.; Di, L.; Zheng, X. Suppressive Effect of Ginsenoside Rg3 against Lipopolysaccharide-Induced Depression-Like Behavior and Neuroinflammation in Mice. J. Agric. Food Chem. 2017, 65, 6861-6869. [CrossRef] [PubMed] 
108. Xu, J.N.; Chen, L.F.; Su, J.; Liu, Z.L.; Chen, J.; Lin, Q.F.; Mao, W.D.; Shen, D. The anxiolytic-like effects of ginsenoside Rg3 on chronic unpredictable stress in rats. Sci. Rep. 2018, 8, 7741. [CrossRef]

109. Zhang, J.Q.; Wu, X.H.; Feng, Y.; Xie, X.F.; Fan, Y.H.; Yan, S.; Zhao, Q.Y.; Peng, C.; You, Z.L. Salvianolic acid B ameliorates depressive-like behaviors in chronic mild stress-treated mice: Involvement of the neuroinflammatory pathway. Acta Pharmacol. Sin. 2016, 37, 1141-1153. [CrossRef]

110. Wang, S.; Wang, C.; Yu, Z.; Wu, C.; Peng, D.; Liu, X.; Liu, Y.; Yang, Y.; Guo, P.; Wei, J. Agarwood Essential Oil Ameliorates Restrain Stress-Induced Anxiety and Depression by Inhibiting HPA Axis Hyperactivity. Int. J. Mol. Sci. 2018, 19, 3468. [CrossRef]

111. Yang, S.J.; Yu, H.Y.; Kang, D.Y.; Ma, Z.Q.; Qu, R.; Fu, Q.; Ma, S.P. Antidepressant-Like effects of salidroside on olfactory bulbectomy-induced pro-inflammatory cytokine production and hyperactivity of HPA axis in rats. Pharmacol. Biochem. Behav. 2014, 124, 451-457. [CrossRef]

112. Zhang, X.; Du, Q.; Liu, C.; Yang, Y.; Wang, J.; Duan, S.; Duan, J. Rhodioloside ameliorates depressive behavior via up-regulation of monoaminergic system activity and anti-inflammatory effect in olfactory bulbectomized rats. Int. Immunopharmacol. 2016, 36, 300-304. [CrossRef] [PubMed]

113. Wu, J.; Du, J.; Xu, C.; Le, J.; Xu, Y.; Liu, B.; Dong, J. Icariin attenuates social defeat-induced down-regulation of glucocorticoid receptor in mice. Pharmacol. Biochem. Behav. 2011, 98, 273-278. [CrossRef] [PubMed]

114. Fan, C.; Song, Q.; Wang, P.; Li, Y.; Yang, M.; Liu, B.; Yu, S.Y. Curcumin Protects Against Chronic Stress-induced Dysregulation of Neuroplasticity and Depression-like Behaviors via Suppressing IL-1 $\beta$ Pathway in Rats. Neuroscience 2018, 392, 92-106. [CrossRef] [PubMed]

115. Zhang, W.Y.; Guo, Y.J.; Han, W.X.; Yang, M.Q.; Wen, L.P.; Wang, K.Y.; Jiang, P. Curcumin relieves depressive-like behaviors via inhibition of the NLRP3 inflammasome and kynurenine pathway in rats suffering from chronic unpredictable mild stress. Int. Immunopharmacol. 2019, 67, 138-144. [CrossRef]

116. Xu, Y.; Ku, B.; Tie, L.; Yao, H.; Jiang, W.; Ma, X.; Li, X. Curcumin reverses the effects of chronic stress on behavior, the HPA axis, BDNF expression and phosphorylation of CREB. Brain Res. 2006, 1122, 56-64. [CrossRef]

117. Wang, F.; Wang, J.; An, J.; Yuan, G.; Hao, X.; Zhang, Y. Resveratrol ameliorates depressive disorder through the NETRIN1-mediated extracellular signal-regulated kinase/cAMP signal transduction pathway. Mol. Med. Rep. 2018, 17, 4611-4618. [CrossRef]

118. Yang, X.H.; Song, S.Q.; Xu, Y. Resveratrol ameliorates chronic unpredictable mild stress-induced depression-like behavior: Involvement of the HPA axis, inflammatory markers, BDNF, and Wnt/ $\beta$-catenin pathway in rats. Neuropsychiatr. Dis. Treat. 2017, 13, 2727-2736. [CrossRef]

119. Li, H.Y.; Zhao, Y.H.; Zeng, M.J.; Fang, F.; Li, M.; Qin, T.T.; Ye, L.Y.; Li, H.W.; Qu, R.; Ma, S.P. Saikosaponin D relieves unpredictable chronic mild stress induced depressive-like behavior in rats: Involvement of HPA axis and hippocampal neurogenesis. Psychopharmacology 2017, 234, 3385-3394. [CrossRef]

120. Guo, L.T.; Wang, S.Q.; Su, J.; Xu, L.X.; Ji, Z.Y.; Zhang, R.Y.; Zhao, Q.W.; Ma, Z.Q.; Deng, X.Y.; Ma, S.P. Baicalin ameliorates neuroinflammation-induced depressive-like behavior through inhibition of toll-like receptor 4 expression via the PI3K/AKT/FoxO1 pathway. J. Neuroinflamm. 2019, 16, 95. [CrossRef]

121. Zhang, C.Y.; Zeng, M.J.; Zhou, L.P.; Li, Y.Q.; Zhao, F.; Shang, Z.Y.; Deng, X.Y.; Ma, Z.Q.; Fu, Q.; Ma, S.P.; et al. Baicalin exerts neuroprotective effects via inhibiting activation of GSK3 $\beta / \mathrm{NF}-\mathrm{kB} / \mathrm{NLRP} 3$ signal pathway in a rat model of depression. Int. Immunopharmacol. 2018, 64, 175-182. [CrossRef]

122. Li, Y.C.; Wang, L.L.; Pei, Y.Y.; Shen, J.D.; Li, H.B.; Wang, B.Y.; Bai, M. Baicalin decreases SGK1 expression in the hippocampus and reverses depressive-like behaviors induced by corticosterone. Neuroscience 2015, 311, 130-137. [CrossRef] [PubMed]

123. Shi, B.; Luo, J.; Fang, Y.; Liu, X.; Rao, Z.; Liu, R.; Zeng, N. Xiaoyao Pills Prevent Lipopolysaccharide-Induced Depression by Inhibiting Inflammation and Protecting Nerves. Front. Pharmacol. 2019, 10, 1324. [CrossRef] [PubMed]

124. Yang, L.; Shergis, J.L.; Di, Y.M.; Zhang, A.L.; Lu, C.; Guo, X.; Fang, Z.; Xue, C.C.; Li, Y. Managing Depression with Bupleurum chinense Herbal Formula: A Systematic Review and Meta-Analysis of Randomized Controlled Trials. J. Altern. Complement. Med. 2020, 26, 8-24. [CrossRef] [PubMed]

125. Yu, Y.; Zhang, G.; Han, T.; Li, J.; Huang, H.L. Network Meta-Analysis of Chinese Patent Medicines in Treatment of Liver Stagnation and Spleen Deficiency of Depression. Zhongguo Zhongyao Zazhi 2019, 44, 5217-5224. [CrossRef]

126. Jing, W.; Song, S.; Sun, H.; Chen, Y.; Zhao, Q.; Zhang, Y.; Dai, G.; Ju, W. Mahuang-Fuzi-Xixin Decoction Reverses Depression-Like Behavior in LPS-Induced Mice by Regulating NLRP3 Inflammasome and Neurogenesis. Neural Plast. 2019, $2019,1571392$. [CrossRef]

127. Du, Y.; Ruan, J.; Zhang, L.; Fu, F. Jieyu Anshen Granule, a Chinese Herbal Formulation, Exerts Effects on Poststroke Depression in Rats. Evid. Based Complement. Altern. Med. 2020, 2020, 7469068. [CrossRef]

128. Guo, F.; Wang, L.; Yang, Y.; Li, L. Clinical observation on treating post-stroke depression with Anshen Jieyu Wan. Clin. J. Chin. Med. 2018, 10. [CrossRef]

129. Zhe, Q.; Sulei, W.; Weiwei, T.; Hongyan, L.; Jianwei, W. Effects of Jiaotaiwan on depressive-like behavior in mice after lipopolysaccharide administration. Metab. Brain Dis. 2017, 32, 415-426. [CrossRef]

130. Li, Y. Clinical observation on application of Jiao Tai Pill on Shenque (CV 8) in treating insomnia. J. Acupunct. Tuina Sci. 2010, 8, 35-37. [CrossRef] 
131. Zhang, Q.; Ma, X.; Yang, Y.; Zhao, H.; Wang, Y.; Yao, X.; Wang, L.; Chang, J.; Zou, H. Jieyuanshen Decoction Exerts Antidepressant Effects on Depressive Rat Model Via Regulating Hpa Axis and the Level of Amino Acids Neurotransmitter. Afr. J. Tradit. Complement. Altern. Med. 2017, 14, 33-46. [CrossRef]

132. Xing, H.; Zhang, K.; Zhang, R.; Shi, H.; Bi, K.; Chen, X. Antidepressant-Like effect of the water extract of the fixed combination of Gardenia jasminoides, Citrus aurantium and Magnolia officinalis in a rat model of chronic unpredictable mild stress. Phytomedicine 2015, 22, 1178-1185. [CrossRef] [PubMed]

133. Chen, L.; Chen, M.; Wang, F.; Sun, Z.; Quanzhi, H.; Geng, M.; Chen, H.; Duan, D. Antidepressant-Like effects of shuyusan in rats exposed to chronic stress: Effects on hypothalamic-pituitary-adrenal function. Evid. Based Complement. Altern. Med. 2012, 2012, 940846. [CrossRef] [PubMed]

134. Li, L.; Yu, A.L.; Wang, Z.L.; Chen, K.; Zheng, W.; Zhou, J.J.; Xie, Q.; Yan, H.B.; Ren, P.; Huang, X. Chaihu-Shugan-San and absorbed meranzin hydrate induce anti-atherosclerosis and behavioral improvements in high-fat diet ApoE ${ }^{-/-}$mice via anti-inflammatory and BDNF-TrkB pathway. Biomed. Pharmacother. 2019, 115, 108893. [CrossRef]

135. Li, Y.H.; Zhang, C.H.; Wang, S.E.; Qiu, J.; Hu, S.Y.; Xiao, G.L. Effects of Chaihu Shugan San on behavior and plasma levels of corticotropin releasing hormone and adrenocorticotropic hormone of rats with chronic mild unpredicted stress depression. Zhong Xi Yi Jie He Xue Bao 2009, 7, 1073-1077. [CrossRef] [PubMed]

136. Wang, Y.; Fan, R.; Huang, X. Meta-Analysis of the clinical effectiveness of traditional Chinese medicine formula Chaihu-ShuganSan in depression. J. Ethnopharmacol. 2012, 141, 571-577. [CrossRef]

137. Sun, Y.; Xu, X.; Zhang, J.; Chen, Y. Treatment of depression with Chai Hu Shu Gan San: A systematic review and meta-analysis of 42 randomized controlled trials. BMC Complement. Altern. Med. 2018, 18, 66. [CrossRef]

138. Cao, C.; Liu, M.; Qu, S.; Huang, R.; Qi, M.; Zhu, Z.; Zheng, J.; Chen, Z.; Wang, Z.; Han, Z.; et al. Chinese medicine formula Kai-Xin-San ameliorates depression-like behaviours in chronic unpredictable mild stressed mice by regulating gut microbiotainflammation-stress system. J. Ethnopharmacol. 2020, 261, 113055. [CrossRef]

139. Bao, Z.; Zhao, G.; Sun, W.; Chen, B. Clinical Curative Effects of Kaixin Powder on Depression with Mild or Moderate Degree. Chin. Arch. Tradit. Chin. Med. 2011, 29. [CrossRef]

140. Cao, C.; Xiao, J.; Liu, M.; Ge, Z.; Huang, R.; Qi, M.; Zhu, H.; Zhu, Y.; Duan, J.A. Active components, derived from Kai-xin-san, a herbal formula, increase the expressions of neurotrophic factor NGF and BDNF on mouse astrocyte primary cultures via cAMP-dependent signaling pathway. J. Ethnopharmacol. 2018, 224, 554-562. [CrossRef]

141. Zhou, J.; Cai, H.; Duan, Y.; Pei, K.; Fan, K.L.; Xu, Y.Y.; Zhao, J.Y.; Liu, J. Research progress on antidepressant effects of Sini San based on three progressive levels of "single herb, herb-pair, and complicated Chinese herbal formula". Zhongguo Zhongyao Zazhi 2018, 43, 46-51. [CrossRef]

142. Bo, P.; Chen, Q.M.; Zhu, H.H.; Zhang, X.D.; Xu, H.R.; Zhang, Y.; Cao, Y.J. Clinical observations on 46 cases of globus hystericus treated with modified Banxia Houpu decoction. J. Tradit. Chin. Med. 2010, 30, 103-107. [CrossRef]

143. Nair, A.B.; Jacob, S. A simple practice guide for dose conversion between animals and human. J. Basic Clin. Pharm. 2016, 7, 27-31. [CrossRef]

144. Juruena, M.F. Early-Life stress and HPA axis trigger recurrent adulthood depression. Epilepsy Behav. 2014, 38, 148-159. [CrossRef] [PubMed]

145. Juruena, M.F.; Agustini, B.; Cleare, A.J.; Young, A.H. A translational approach to clinical practice via stress-responsive glucocorticoid receptor signaling. Stem Cell Investig. 2017, 4, 13. [CrossRef]

146. Pandey, G.N.; Rizavi, H.S.; Bhaumik, R.; Ren, X. Increased protein and mRNA expression of corticotropin-releasing factor $(\mathrm{CRF})$, decreased CRF receptors and CRF binding protein in specific postmortem brain areas of teenage suicide subjects. Psychoneuroendocrinology 2019, 106, 233-243. [CrossRef] [PubMed]

147. Horchar, M.J.; Wohleb, E.S. Glucocorticoid receptor antagonism prevents microglia-mediated neuronal remodeling and behavioral despair following chronic unpredictable stress. Brain Behav. Immun. 2019, 81, 329-340. [CrossRef]

148. Pariante, C.M.; Lightman, S.L. The HPA axis in major depression: Classical theories and new developments. Trends Neurosci. 2008, 31, 464-468. [CrossRef]

149. Gillespie, C.F.; Nemeroff, C.B. Hypercortisolemia and depression. Psychosom. Med. 2005, 67, S26-S28. [CrossRef]

150. D'Alessio, L.; Mesarosova, L.; Anink, J.J.; Kochen, S.; Solis, P.; Oddo, S.; Konopka, H.; Lyer, A.M.; Mühlebner, A.; Lucassen, P.J.; et al. Reduced expression of the glucocorticoid receptor in the hippocampus of patients with drug-resistant temporal lobe epilepsy and comorbid depression. Epilepsia 2020, 61, 1595-1605. [CrossRef]

151. Waters, R.P.; Rivalan, M.; Bangasser, D.A.; Deussing, J.M.; Ising, M.; Wood, S.K.; Holsboer, F.; Summers, C.H. Evidence for the role of corticotropin-releasing factor in major depressive disorder. Neurosci. Biobehav. Rev. 2015, 58, 63-78. [CrossRef]

152. Fahmy, H.; Kuppast, B.; Ismail, M.T. Structure and Function of Small Non-Peptide CRF Antagonists and Their Potential Clinical Use. Curr. Mol. Pharmacol. 2017, 10, 270-281. [CrossRef]

153. Bhutada, P.; Mundhada, Y.; Bansod, K.; Ubgade, A.; Quazi, M.; Umathe, S.; Mundhada, D. Reversal by quercetin of corticotrophin releasing factor induced anxiety- and depression-like effect in mice. Prog. Neuropsychopharmacol. Biol. Psychiatry 2010, 34, 955-960. [CrossRef] [PubMed]

154. Kawabata, K.; Kawai, Y.; Terao, J. Suppressive effect of quercetin on acute stress-induced hypothalamic-pituitary-adrenal axis response in Wistar rats. J. Nutr. Biochem. 2010, 21, 374-380. [CrossRef] 
155. Ketchesin, K.D.; Stinnett, G.S.; Seasholtz, A.F. Corticotropin-Releasing hormone-binding protein and stress: From invertebrates to humans. Stress 2017, 20, 449-464. [CrossRef]

156. Nielsen, D.M. Corticotropin-Releasing factor type-1 receptor antagonists: The next class of antidepressants? Life Sci. 2006, 78, 909-919. [CrossRef]

157. Henckens, M.J.; Deussing, J.M.; Chen, A. Region-Specific roles of the corticotropin-releasing factor-urocortin system in stress. Nat. Rev. Neurosci. 2016, 17, 636-651. [CrossRef]

158. Im, E. Multi-Facets of Corticotropin-Releasing Factor in Modulating Inflammation and Angiogenesis. J. Neurogastroenterol. Motil. 2015, 21, 25-32. [CrossRef]

159. Dedic, N.; Chen, A.; Deussing, J.M. The CRF Family of Neuropeptides and their Receptors-Mediators of the Central Stress Response. Curr. Mol. Pharmacol. 2018, 11, 4-31. [CrossRef]

160. Paez-Pereda, M.; Hausch, F.; Holsboer, F. Corticotropin releasing factor receptor antagonists for major depressive disorder. Expert Opin. Investig. Drugs 2011, 20,519-535. [CrossRef]

161. Menke, A. Is the HPA Axis as Target for Depression Outdated, or Is There a New Hope? Front. Psychiatry 2019, 10, 101. [CrossRef]

162. Jutkiewicz, E.M.; Wood, S.K.; Houshyar, H.; Hsin, L.W.; Rice, K.C.; Woods, J.H. The effects of CRF antagonists, antalarmin, CP154,526, LWH234, and R121919, in the forced swim test and on swim-induced increases in adrenocorticotropin in rats. Psychopharmacology 2005, 180, 215-223. [CrossRef]

163. Simmen, U.; Bobirnac, I.; Ullmer, C.; Lübbert, H.; Berger Büter, K.; Schaffner, W.; Schoeffter, P. Antagonist effect of pseudohypericin at CRF1 receptors. Eur. J. Pharmacol. 2003, 458, 251-256. [CrossRef]

164. Thiagarajah, A.S.; Eades, L.E.; Thomas, P.R.; Guymer, E.K.; Morand, E.F.; Clarke, D.M.; Leech, M. GILZ: Glitzing up our understanding of the glucocorticoid receptor in psychopathology. Brain Res. 2014, 1574, 60-69. [CrossRef]

165. Leistner, C.; Menke, A. How to measure glucocorticoid receptor's sensitivity in patients with stress-related psychiatric disorders. Psychoneuroendocrinology 2018, 91, 235-260. [CrossRef]

166. Moraitis, A.G.; Block, T.; Nguyen, D.; Belanoff, J.K. The role of glucocorticoid receptors in metabolic syndrome and psychiatric illness. J. Steroid Biochem. Mol. Biol. 2017, 165, 114-120. [CrossRef]

167. Sarubin, N.; Hilbert, S.; Naumann, F.; Zill, P.; Wimmer, A.M.; Nothdurfter, C.; Rupprecht, R.; Baghai, T.C.; Bühner, M.; Schüle, C. The sex-dependent role of the glucocorticoid receptor in depression: Variations in the NR3C1 gene are associated with major depressive disorder in women but not in men. Eur. Arch. Psychiatry Clin. Neurosci. 2017, 267, 123-133. [CrossRef]

168. Pandey, G.N.; Rizavi, H.S.; Ren, X.; Dwivedi, Y.; Palkovits, M. Region-Specific alterations in glucocorticoid receptor expression in the postmortem brain of teenage suicide victims. Psychoneuroendocrinology 2013, 38, 2628-2639. [CrossRef]

169. Guidotti, G.; Calabrese, F.; Anacker, C.; Racagni, G.; Pariante, C.M.; Riva, M.A. Glucocorticoid receptor and FKBP5 expression is altered following exposure to chronic stress: Modulation by antidepressant treatment. Neuropsychopharmacology 2013, 38, 616-627. [CrossRef]

170. Van Rossum, E.F.; Binder, E.B.; Majer, M.; Koper, J.W.; Ising, M.; Modell, S.; Salyakina, D.; Lamberts, S.W.; Holsboer, F. Polymorphisms of the glucocorticoid receptor gene and major depression. Biol. Psychiatry 2006, 59, 681-688. [CrossRef]

171. Block, T.S.; Kushner, H.; Kalin, N.; Nelson, C.; Belanoff, J.; Schatzberg, A. Combined Analysis of Mifepristone for Psychotic Depression: Plasma Levels Associated with Clinical Response. Biol. Psychiatry 2018, 84, 46-54. [CrossRef]

172. Soria, V.; Gonzalez-Rodriguez, A.; Huerta-Ramos, E.; Usall, J.; Cobo, J.; Bioque, M.; Barbero, J.D.; García-Rizo, C.; Tost, M.; Monreal, J.A.; et al. Targeting hypothalamic-pituitary-adrenal axis hormones and sex steroids for improving cognition in major mood disorders and schizophrenia: A systematic review and narrative synthesis. Psychoneuroendocrinology 2018, 93, 8-19. [CrossRef]

173. Wang, W.; Liu, L.; Yang, X.; Gao, H.; Tang, Q.K.; Yin, L.Y.; Yin, X.Y.; Hao, J.R.; Geng, D.Q.; Gao, C. Ketamine improved depressivelike behaviors via hippocampal glucocorticoid receptor in chronic stress induced-susceptible mice. Behav. Brain Res. 2019, 364, 75-84. [CrossRef]

174. Garde, D. Corcept Tanks as Depression Drug Comes Up Short In Phase III. Available online: https:/ /www.fiercebiotech.com/ biotech/corcept-tanks-as-depression-drug-comes-up-short-phase-iii (accessed on 5 May 2014).

175. Clark, R.D. Glucocorticoid receptor antagonists. Curr. Top. Med. Chem. 2008, 8, 813-838. [CrossRef]

176. Luo, Y.; Yang, M.; Guo, M.; Zhong, X.; Hu, Y. Huang Qin Hua Shi decoction for high-temperature- and high-humidity-induced cognitive-behavioral disorder in rats is associated with deactivation of the hypothalamic-pituitary-adrenal axis. J. Int. Med. Res. 2019, 47, 5752-5766. [CrossRef]

177. Horowitz, M.A.; Cattaneo, A.; Cattane, N.; Lopizzo, N.; Tojo, L.; Bakunina, N.; Musaelyan, K.; Borsini, A.; Zunszain, P.A.; Pariante, C.M. Glucocorticoids prime the inflammatory response of human hippocampal cells through up-regulation of inflammatory pathways. Brain Behav. Immun. 2020, 87, 777-794. [CrossRef]

178. Bottasso, E. Toward the Existence of a Sympathetic Neuroplasticity Adaptive Mechanism Influencing the Immune Response. A Hypothetical View-Part II. Front. Endocrinol. 2019, 10, 633. [CrossRef]

179. Dai, S.; Mo, Y.; Wang, Y.; Xiang, B.; Liao, Q.; Zhou, M.; Li, X.; Li, Y.; Xiong, W.; Li, G.; et al. Chronic Stress Promotes Cancer Development. Front. Oncol. 2020, 10, 1492. [CrossRef]

180. Chrousos, G.P.; Gold, P.W. The concepts of stress and stress system disorders. Overview of physical and behavioral homeostasis. J. Am. Med. Assoc. 1992, 267, 1244-1252. [CrossRef]

181. Chrousos, G.P. Stress and disorders of the stress system. Nat. Rev. Endocrinol. 2009, 5, 374-381. [CrossRef] 
182. Bellavance, M.A.; Rivest, S. The HPA-Immune Axis and the Immunomodulatory Actions of Glucocorticoids in the Brain. Front. Immunol. 2014, 5, 136. [CrossRef]

183. Dantzer, R. Neuroimmune Interactions: From the Brain to the Immune System and Vice Versa. Physiol. Rev. 2018, 98, 477-504. [CrossRef] [PubMed]

184. Newton, R.; Holden, N.S. Separating transrepression and transactivation: A distressing divorce for the glucocorticoid receptor? Mol. Pharmacol. 2007, 72, 799-809. [CrossRef] [PubMed]

185. De Bosscher, K.; Vanden Berghe, W.; Haegeman, G. The interplay between the glucocorticoid receptor and nuclear factor- $\mathrm{kB}$ or activator protein-1: Molecular mechanisms for gene repression. Endocr. Rev. 2003, 24, 488-522. [CrossRef]

186. Bekhbat, M.; Rowson, S.A.; Neigh, G.N. Checks and balances: The glucocorticoid receptor and NFkB in good times and bad. Front. Neuroendocrinol. 2017, 46, 15-31. [CrossRef] [PubMed]

187. Wang, D.; Lin, W.; Pan, Y.; Kuang, X.; Qi, X.; Sun, H. Chronic blockade of glucocorticoid receptors by RU486 enhances lipopolysaccharide-induced depressive-like behaviour and cytokine production in rats. Brain Behav. Immun. 2011, 25, 706-714. [CrossRef]

188. Troubat, R.; Barone, P.; Leman, S.; Desmidt, T.; Cressant, A.; Atanasova, B.; Brizard, B.; Hage, W.E.; Surget, A.; Belzung, C.; et al. Neuroinflammation and depression: A review. Eur. J. Neurosci. 2020. [CrossRef] [PubMed]

189. Li, K.D.; Yan, K.; Wang, Q.S.; Tian, J.S.; Xu, D.; Zhang, W.Y.; Cui, Y.L. Antidepressant-Like effects of dietary gardenia blue pigment derived from genipin and tyrosine. Food Funct. 2019, 10, 4533-4545. [CrossRef]

190. Dunn, A.J. Effects of cytokines and infections on brain neurochemistry. Clin. Neurosci. Res. 2006, 6, 52-68. [CrossRef]

191. Chen, H.; Shi, H.; Liu, Y.; Ren, X.; He, S.; Chang, X.; Yin, Y. Activation of corticotropin-releasing factor receptor 1 aggravates dextran sodium sulphate-induced colitis in mice by promoting M1 macrophage polarization. Mol. Med. Rep. 2018, 17, $234-242$. [CrossRef]

192. Knapp, D.J.; Whitman, B.A.; Wills, T.A.; Angel, R.A.; Overstreet, D.H.; Criswell, H.E.; Ming, Z.; Breese, G.R. Cytokine involvement in stress may depend on corticotrophin releasing factor to sensitize ethanol withdrawal anxiety. Brain Behav. Immun. 2011, 25, S146-S154. [CrossRef]

193. Chatoo, M.; Li, Y.; Ma, Z.; Coote, J.; Du, J.; Chen, X. Involvement of Corticotropin-Releasing Factor and Receptors in Immune Cells in Irritable Bowel Syndrome. Front. Endocrinol. 2018, 9, 21. [CrossRef] [PubMed]

194. Frank, M.G.; Weber, M.D.; Watkins, L.R.; Maier, S.F. Stress sounds the alarmin: The role of the danger-associated molecular pattern HMGB1 in stress-induced neuroinflammatory priming. Brain Behav. Immun. 2015, 48, 1-7. [CrossRef] [PubMed]

195. Alvarez-Carbonell, D.; Ye, F.; Ramanath, N.; Dobrowolski, C.; Karn, J. The Glucocorticoid Receptor Is a Critical Regulator of HIV Latency in Human Microglial Cells. J. Neuroimmune Pharmacol. 2019, 14, 94-109. [CrossRef] [PubMed]

196. Wang, W.; Ji, P.; Dow, K.E. Corticotropin-Releasing hormone induces proliferation and TNF- $\alpha$ release in cultured rat microglia via MAP kinase signalling pathways. J. Neurochem. 2003, 84, 189-195. [CrossRef] [PubMed]

197. Herrera, A.J.; Espinosa-Oliva, A.M.; Carrillo-Jimenez, A.; Oliva-Martin, M.J.; Garcia-Revilla, J.; Garcia-Quintanilla, A.; Pablos, R.M.; Venero, J.L. Relevance of chronic stress and the two faces of microglia in Parkinson's disease. Front. Cell. Neurosci. 2015, 9 , 312. [CrossRef] [PubMed]

198. Kato, T.A.; Hayakawa, K.; Monji, A.; Kanba, S. Missing and Possible Link between Neuroendocrine Factors, Neuropsychiatric Disorders, and Microglia. Front. Integr. Neurosci. 2013, 7, 53. [CrossRef]

199. Raison, C.L.; Borisov, A.S.; Woolwine, B.J.; Massung, B.; Vogt, G.; Miller, A.H. Interferon- $\alpha$ effects on diurnal hypothalamicpituitary-adrenal axis activity: Relationship with proinflammatory cytokines and behavior. Mol. Psychiatry 2010, 15, 535-547. [CrossRef]

200. Panossian, A.; Seo, E.J.; Efferth, T. Novel molecular mechanisms for the adaptogenic effects of herbal extracts on isolated brain cells using systems biology. Phytomedicine 2018, 50, 257-284. [CrossRef]

201. Panossian, A.G.; Efferth, T.; Shikov, A.N.; Pozharitskaya, O.N.; Kuchta, K.; Mukherjee, P.K.; Banerjee, S.; Heinrich, M.; Wu, W.; Guo, D.A.; et al. Evolution of the adaptogenic concept from traditional use to medical systems: Pharmacology of stress- and aging-related diseases. Med. Res. Rev. 2021, 41, 630-703. [CrossRef]

202. Amsterdam, J.D.; Panossian, A.G. Rhodiola rosea L. as a putative botanical antidepressant. Phytomedicine 2016, $23,770-783$. [CrossRef]

203. Fu, H.; Xu, Z.; Zhang, X.L.; Zheng, G.Q. Kaixinsan, a Well-Known Chinese Herbal Prescription, for Alzheimer's Disease and Depression: A Preclinical Systematic Review. Front. Neurosci. 2019, 13, 1421. [CrossRef] [PubMed]

204. Hu, Y.; Liu, X.; Zhang, T.; Chen, C.; Dong, X.; Can, Y.; Liu, P. Behavioral and Biochemical Effects of KXS on Postmyocardial Infarction Depression. Front. Pharmacol. 2020, 11, 561817. [CrossRef] [PubMed]

205. Dang, H.; Sun, L.; Liu, X.; Peng, B.; Wang, Q.; Jia, W.; Chen, Y.; Pan, A.; Xiao, P. Preventive action of Kai Xin San aqueous extract on depressive-like symptoms and cognition deficit induced by chronic mild stress. Exp. Biol. Med. 2009, 234, 785-793. [CrossRef] [PubMed]

206. Li, Y.; Wang, L.; Wang, P.; Fan, C.; Zhang, P.; Shen, J.; Yu, S.Y. Ginsenoside-Rg1 Rescues Stress-Induced Depression-Like Behaviors via Suppression of Oxidative Stress and Neural Inflammation in Rats. Oxid. Med. Cell. Longev. 2020, 2020, 2325391. [CrossRef] [PubMed]

207. Lee, K.J.; Ji, G.E. The effect of fermented red ginseng on depression is mediated by lipids. Nutr. Neurosci. 2014, 17, 7-15. [CrossRef] 
208. Lee, Y.Y.; Park, J.S.; Lee, E.J.; Lee, S.Y.; Kim, D.H.; Kang, J.L.; Kim, H.S. Anti-Inflammatory mechanism of ginseng saponin metabolite Rh3 in lipopolysaccharide-stimulated microglia: Critical role of $5^{\prime}$-adenosine monophosphate-activated protein kinase signaling pathway. J. Agric. Food Chem. 2015, 63, 3472-3480. [CrossRef]

209. Ke, L.; Guo, W.; Xu, J.; Zhang, G.; Wang, W.; Huang, W. Ginsenoside Rb1 attenuates activated microglia-induced neuronal damage. Neural Regen. Res. 2014, 9, 252-259. [CrossRef]

210. Chen, S.; Li, X.; Wang, Y.; Mu, P.; Chen, C.; Huang, P.; Liu, D. Ginsenoside Rb1 attenuates intestinal ischemia/reperfusion-induced inflammation and oxidative stress via activation of the PI3K/Akt/Nrf2 signaling pathway. Mol. Med. Rep. 2019, 19, 3633-3641. [CrossRef]

211. Shi, Y.; Wang, H.; Zheng, M.; Xu, W.; Yang, Y.; Shi, F. Ginsenoside Rg3 suppresses the NLRP3 inflammasome activation through inhibition of its assembly. FASEB J. 2020, 34, 208-221. [CrossRef] [PubMed]

212. Lee, W.; Cho, S.H.; Kim, J.E.; Lee, C.; Lee, J.H.; Baek, M.C.; Song, G.Y.; Bae, J.S. Suppressive Effects of Ginsenoside Rh1 on HMGB1-Mediated Septic Responses. Am. J. Chin. Med. 2019, 47, 119-133. [CrossRef]

213. Kim, Y.; Lee, H.Y.; Choi, Y.J.; Cho, S.H. Antidepressant effects of ginsenoside Rf on behavioral change in the glial degeneration model of depression by reversing glial loss. J. Ginseng Res. 2020, 44, 603-610. [CrossRef] [PubMed]

214. Li, Z.; Zhao, L.; Chen, J.; Liu, C.; Li, S.; Hua, M.; Qu, D.; Shao, Z.; Sun, Y. Ginsenoside Rk1 alleviates LPS-induced depression-like behavior in mice by promoting BDNF and suppressing the neuroinflammatory response. Biochem. Biophys. Res. Commun. 2020, 530, 658-664. [CrossRef] [PubMed]

215. Zhang, Y.Q.; Wang, X.B.; Xue, R.R.; Gao, X.X.; Li, W. Ginsenoside Rg1 attenuates chronic unpredictable mild stress-induced depressive-like effect via regulating NF-kB/NLRP3 pathway in rats. Neuroreport 2019, 30, 893-900. [CrossRef] [PubMed]

216. Hu, Y.; Liu, M.; Liu, P.; Guo, D.H.; Wei, R.B.; Rahman, K. Possible mechanism of the antidepressant effect of 3,6'-disinapoyl sucrose from Polygala tenuifolia Willd. J. Pharm. Pharmacol. 2011, 63, 869-874. [CrossRef] [PubMed]

217. Hu, Y.; Liu, P.; Guo, D.H.; Rahman, K.; Wang, D.X.; Xie, T.T. Antidepressant effects of the extract YZ-50 from Polygala tenuifolia in chronic mild stress treated rats and its possible mechanisms. Pharm. Biol. 2010, 48, 794-800. [CrossRef]

218. Liu, P.; Hu, Y.; Guo, D.H.; Wang, D.X.; Tu, H.H.; Ma, L.; Xie, T.T.; Kong, L.Y. Potential antidepressant properties of Radix Polygalae (Yuan Zhi). Phytomedicine 2010, 17, 794-799. [CrossRef]

219. Lee, S.R.; Lee, S.; Moon, E.; Park, H.J.; Park, H.B.; Kim, K.H. Bioactivity-Guided isolation of anti-inflammatory triterpenoids from the sclerotia of Poria cocos using LPS-stimulated Raw264.7 cells. Bioorganic Chem. 2017, 70, 94-99. [CrossRef]

220. Chang, W.; Teng, J. $\beta$-asarone prevents A $\beta 25$-35-induced inflammatory responses and autophagy in SH-SY5Y cells: Down expression Beclin-1, LC3B and up expression Bcl-2. Int. J. Clin. Exp. Med. 2015, 8, 20658-20663.

221. Chen, C.; Hu, Y.; Dong, X.Z.; Zhou, X.J.; Mu, L.H.; Liu, P. Proteomic Analysis of the Antidepressant Effects of Shen-Zhi-Ling in Depressed Patients: Identification of Proteins Associated with Platelet Activation and Lipid Metabolism. Cell. Mol. Neurobiol. 2018, 38, 1123-1135. [CrossRef]

222. Dong, X.Z.; Wang, D.X.; Zhang, T.Y.; Liu, X.; Liu, P.; Hu, Y. Identification of protein targets for the antidepressant effects of Kai-Xin-San in Chinese medicine using isobaric tags for relative and absolute quantitation. Neural Regen. Res. 2020, 15, 302-310. [CrossRef]

223. Zhao, S.; Iyengar, R. Systems pharmacology: Network analysis to identify multiscale mechanisms of drug action. Annu. Rev. Pharmacol. Toxicol. 2012, 52, 505-521. [CrossRef] [PubMed]

224. Woo, H.I.; Lim, S.W.; Myung, W.; Kim, D.K.; Lee, S.Y. Differentially expressed genes related to major depressive disorder and antidepressant response: Genome-Wide gene expression analysis. Exp. Mol. Med. 2018, 50, 92. [CrossRef] [PubMed]

225. Panossian, A.; Hamm, R.; Wikman, G.; Efferth, T. Mechanism of action of Rhodiola, salidroside, tyrosol and triandrin in isolated neuroglial cells: An interactive pathway analysis of the downstream effects using RNA microarray data. Phytomedicine 2014, 21, 1325-1348. [CrossRef] [PubMed]

226. Panossian, A.; Seo, E.J.; Wikman, G.; Efferth, T. Synergy assessment of fixed combinations of Herba Andrographidis and Radix Eleutherococci extracts by transcriptome-wide microarray profiling. Phytomedicine 2015, 22, 981-992. [CrossRef] [PubMed]

227. Panossian, A.; Seo, E.-J.; Efferth, T. Synergy assessments of plant extracts used in the treatment of stress and aging-related disorders. Synergy 2018, 7, 39-48. [CrossRef]

228. Panossian, A.; Hamm, R.; Kadioglu, O.; Wikman, G.; Efferth, T. Synergy and Antagonism of Active Constituents of ADAPT-232 on Transcriptional Level of Metabolic Regulation of Isolated Neuroglial Cells. Front. Neurosci. 2013, 7, 16. [CrossRef] [PubMed]

229. Wang, B.; Lu, S.; Zhang, C.; Zhu, L.; Li, Y.; Bai, M.; Xu, E. Quantitative proteomic analysis of the liver reveals antidepressant potential protein targets of Sinisan in a mouse CUMS model of depression. Biomed. Pharmacother. 2020, 130, 110565. [CrossRef] 